\title{
Losses and depolarization of ultracold neutrons on neutron guide and storage materials
}

\author{
V. Bondar, ${ }^{1,9, *}$ S. Chesnevskaya,${ }^{2, \dagger}$ M. Daum,,${ }^{1,}$ B. Franke, ${ }^{1,5,8}$ P. Geltenbort,${ }^{4}$ L. Göltl, ${ }^{1}$ E. Gutsmiedl, ${ }^{2}$ J. Karch,${ }^{3}$ \\ M. Kasprzak, ${ }^{6, \|}$ G. Kessler, ${ }^{2}$ K. Kirch, ${ }^{1,5}$ H.-C. Koch, ${ }^{3,}$, A. Kraft, ${ }^{3}$ T. Lauer, ${ }^{3,}{ }^{, *}$ B. Lauss, ${ }^{1}$ E. Pierre, ${ }^{1,7, \dagger \dagger}$ G. Pignol, ${ }^{8}$ \\ D. Reggiani, ${ }^{1}$ P. Schmidt-Wellenburg, ${ }^{1}$ Yu. Sobolev, ${ }^{3}$ T. Zechlau, ${ }^{3, \ddagger \ddagger}$ and G. Zsigmond ${ }^{1}$ \\ ${ }^{1}$ Paul Scherrer Institute (PSI), CH-5232 Villigen PSI, Switzerland \\ ${ }^{2}$ Physik Department, Technische Universität München (TUM), Exzellenzcluster Universe, D-85748 Garching, Germany \\ ${ }^{3}$ Institut für Physik, Johannes Gutenberg University, 55099 Mainz, Germany \\ ${ }^{4}$ Institut Laue-Langevin (ILL), 38000 Grenoble, France \\ ${ }^{5}$ IPP, Institut für Teilchenphysik, ETH Zürich, CH-8093 Zürich, Switzerland \\ ${ }^{6}$ Physics Department, University of Fribourg, CH-1700 Fribourg, Switzerland \\ ${ }^{7}$ Laboratoire de Physique Corpusculaire (LPC), ENSICAEN-CNRS/IN2P3, Caen, France \\ ${ }^{8}$ Laboratoire de Physique Subatomique et de Cosmologie, Université Grenoble-Alpes, CNRS/IN2P3, Grenoble, France \\ ${ }^{9}$ Kyiv Polytechnic Institute (KPI), Kyiv, Ukraine, 03056 \\ (Received 3 May 2017; published 19 September 2017)
}

\begin{abstract}
At Institut Laue-Langevin (ILL) and Paul Scherrer Institute (PSI), we have measured the losses and depolarization probabilities of ultracold neutrons on various materials: (i) nickel-molybdenum alloys with weight percentages of 82/18,85/15,88/12, 91/9, and 94/6 and natural nickel Ni100, (ii) nickel-vanadium NiV93/7, (iii) copper, and (iv) deuterated polystyrene (dPS). For the different samples, storage-time constants up to $\sim 460 \mathrm{~s}$ were obtained at room temperature. The corresponding loss parameters for ultracold neutrons, $\eta$, varied between $1.0 \times 10^{-4}$ and $2.2 \times 10^{-4}$. All $\eta$ values are in agreement with theory except for dPS, where anomalous losses at room temperature were established with four standard deviations. The depolarization probabilities per wall collision $\beta$ measured with unprecedented sensitivity varied between $0.7 \times 10^{-6}$ and $9.0 \times 10^{-6}$. Our depolarization result for copper differs from other experiments by 4.4 and 15.8 standard deviations. The $\beta$ values of the paramagnetic NiMo alloys over molybdenum content show an increase of $\beta$ with increasing Mo content. This is in disagreement with expectations from literature. Finally, ferromagnetic behavior of NiMo alloys at room temperature was found for molybdenum contents of 6.5 at.\% or less and paramagnetic behavior for more than 8.7 at.\%. This may contribute to solving an ambiguity in literature.
\end{abstract}

DOI: 10.1103/PhysRevC.96.035205

\section{INTRODUCTION}

A nonzero electric dipole moment of the neutron (nEDM) does not conserve both parity, $\mathcal{P}$, and time reversal, $\mathcal{T}$, symmetries [1-3] and, because of the $\mathcal{C P} \mathcal{T}$ theorem [4], also does not conserve the combined charge and parity symmetry, $\mathcal{C P}$. In order to explain the observed baryon asymmetry in the Universe, additional $\mathcal{C P}$ violation beyond that established in

\footnotetext{
*Present address: University of Leuven, Leuven, Belgium.

${ }^{\dagger}$ Present address: Extreme Light Infrastructure-Nuclear Physics (ELI-NP), ul. Reactorului no. 30, P. O. Box MG-6, BucharestMagurele, 077125, Romania.

${ }^{\ddagger}$ Corresponding author: manfred.daum@psi.ch

${ }^{\S}$ Present address: TRIUMF, 4004 Wesbrook Mall, Vancouver, BC V6T 2A3, Canada.

"Present address: University of Leuven, Leuven, Belgium.

"Present address: Paul Scherrer Institute (PSI), CH-5232 Villigen PSI, Switzerland.

${ }^{* *}$ Present address: Movatec GmbH, Erfurter Str. 23, 85386 Eching, Germany.

${ }^{\dagger}$ Present addresses: TRIUMF, 4004 Wesbrook Mall, Vancouver, BC V6T 2A3, Canada, and RCNP, Research Center for Nuclear Physics, Osaka University, Osaka, Japan.

${ }_{\ddagger}^{\ddagger}$ Present address: Movatec GmbH, Erfurter Str. 23, 85386 Eching, Germany.
}

the standard model of particle physics is necessary [5,6]. The nEDM is among the most sensitive probes for such additional sources of $\mathcal{C P}$ nonconservation. The experiment currently in operation at the Paul Scherrer Institute (PSI) [7,8] is an upgrade of the Rutherford-Sussex-Institut Laue-Langevin (ILL) experiment $[9,10]$ with the most recent result presented in Ref. [11].

Besides many tools for the suppression of systematic uncertainties in the experiment at PSI [7], there are mainly five items to improve the overall sensitivity of the measurement:

(1) The total number of neutrons, which is permanently increased by improving the intensity of the present source of ultracold neutrons (UCN) ${ }^{1}$ at PSI [15-19]. This source is based on a very cold moderator-converter using solid deuterium $\left(\mathrm{sD}_{2}\right)$ [20-33] at a temperature around $5 \mathrm{~K}$.

(2) An increase of the electric field, $E$.

(3) An increase of the storage time (or the time of spin precession).

\footnotetext{
${ }^{1}$ Ultracold neutrons (UCN) are very slow neutrons with velocities $v_{\mathrm{n}} \leqslant 7 \mathrm{~m} / \mathrm{s}$. They can undergo total reflection at a material surface [12] and can be stored in bottles with long observation times of many minutes $[13,14]$.
} 
(4) The initial polarization of the neutrons, which is increased to $\sim 100 \%$ by means of a superconductive solenoid with a field of $5 \mathrm{~T}$ [34].

(5) The simultaneous measurement of both spin states $[35,36]$.

In order to minimize the influence of the high magnetic field $(5 \mathrm{~T})$ of the neutron polarizer on the spin precession in the nEDM experiment, the neutron polarizer must be shielded or compensated and placed at some distance $(\sim 2$ to $5 \mathrm{~m}$ ) from the chamber for the precession of the neutron spin. The neutron guides linking the neutron polarizer with this chamber as well as those guides linking the chamber with the detectors should minimize depolarization and losses of neutrons. They have to fulfill the following criteria:

(1) Minimal surface roughness to reduce non-specular reflections. This is achieved by using glass tubes with a roughness at the inner surface of less than $\sim 2 \mathrm{~nm}$ as substrate for coatings for the reflection of UCN.

(2) High optical potential of the inner walls of the guides. It is often a useful approximation to consider the velocity spectrum of a UCN source to be Maxwellian [37]. Then, the low-energy part of the spectrum follows a $v^{2}$ dependence and its integral is proportional to $v^{3}$ or $E_{\mathrm{kin}}^{3 / 2}$ with the maximum of $E_{\text {kin }} \approx V_{\mathrm{f}}$, where $V_{\mathrm{f}}$ is the optical potential (sometimes also termed Fermi potential) of the UCN guide. The glass tubes were coated inside with $500 \mathrm{~nm}$ of different materials under investigation; see Table I. They were selected for high $V_{\mathrm{f}}$.

TABLE I. Different coatings for the investigation of UCN losses and depolarization. NiMo, nickel-molybdenum alloy; NiV, nickelvanadium alloy; $\mathrm{Ni}, \mathrm{Cu}$, natural nickel and natural copper; and dPS, deuterated polystyrene. The samples were glass tubes (with the exception of dPS, which was coated on a PS tube) with length $1500 \mathrm{~mm}$, outer diameter $80 \mathrm{~mm}$, inner diameter $70 \mathrm{~mm}$, and surface roughness $\sim 2 \mathrm{~nm}$ coated at the inner surface with thicknesses around $500 \mathrm{~nm}$. The real part, $V_{\mathrm{f}}$, and the imaginary part, $W$, of the optical potentials were calculated according to Refs. [37-41]. Flat probes sputtered at the same sputtering facility as the tube samples were measured at PSI using cold neutron reflectometry [42]. The results for $V_{\mathrm{f}}$ were found in agreement with the calculated values within uncertainties, $\Delta V_{\mathrm{f}}= \pm 10 \mathrm{neV}$. For the ferromagnetic samples, the average optical potential (in brackets) is reduced due to magnetization in the strong magnetic field; see Refs. [43-46].

\begin{tabular}{lcccc}
\hline \hline Material & $\begin{array}{c}\text { weigth } \\
\text { percentages } \\
\text { [wt.\%] }\end{array}$ & $\begin{array}{c}\text { Atomic } \\
\text { percentages } \\
\text { [at.\%] }\end{array}$ & \multicolumn{1}{c}{$V_{\mathrm{f}}$} & $W$ \\
\hline NiMo & $82 / 18$ & $86.6 / 13.4$ & 221.5 & 0.027 \\
NiMo & $85 / 15$ & $89.0 / 11.0$ & 225.4 & 0.027 \\
NiMo & $88 / 12$ & $91.3 / 8.7$ & 229.5 & 0.028 \\
NiMo & $91 / 9$ & $93.5 / 6.5$ & $223.5(233.6)$ & 0.028 \\
NiMo & $94 / 6$ & $95.7 / 4.3$ & $218.7(237.5)$ & 0.029 \\
Ni & $100 / 0$ & $100.0 / 0.0$ & $209.5(245.5)$ & 0.030 \\
NiV & $93 / 7$ & $94.4 / 5.6$ & $210.1(227.0)$ & 0.030 \\
$\mathrm{Cu}$ & $100 / 0$ & $100.0 / 0.0$ & 167 & 0.026 \\
$\mathrm{dPS}$ & $100 / 0$ & $100.0 / 0.0$ & 161 & 0.0003 \\
\hline \hline
\end{tabular}

(3) Minimal loss probability per wall collision.

(4) Minimal depolarization probability per wall collision.

Items 1 and 2 have already been investigated in Ref. [47]; items 3 and 4 are the objectives of the present investigation.

\section{UCN LOSSES AND DEPOLARIZATION FROM SURFACES}

The correlation between the optical potential and UCN losses is beautifully described in Refs. [37,41]. Here, we present a short outline in order to make the paper selfcontained. For further reading, see also Refs. [48,49].

The optical potential of a material is complex, $U=V_{\mathrm{f}}-$ $i W$, where $V_{\mathrm{f}}$ and $W$ depend on the nuclear properties of the surface atoms:

$$
V_{\mathrm{f}}=\frac{2 \pi \hbar^{2}}{m} a b ; \quad W=\frac{\hbar}{2} a \sigma v .
$$

Here, $m$ denotes the neutron mass, $a$ is the scattering center density, $b$ is the bound coherent nuclear scattering length, $\sigma$ is the loss cross section per nucleus of the surface material, and $v$ is the velocity of the neutron. For neutrons reflecting at a surface, the real part of the potential, $V_{\mathrm{f}}$, sets the critical velocity, $v_{c}=\sqrt{2 V_{\mathrm{f}} / m}$, for total reflection and the imaginary part, $W$, determines the reflection loss, $\eta$ [37]:

$$
\eta=W / V_{\mathrm{f}}=(\sigma m v) /(4 \pi \hbar b)
$$

Since $\sigma$ is proportional to $1 / v$, the parameter $\eta$ is independent of neutron kinetic energy.

The loss rate, $1 / \tau_{\mu}$, originating from wall interactions for UCN of one specific kinetic energy, $E$, and without the influence of external fields, is given by

$$
\frac{1}{\tau_{\mu}}=\mu(\theta, E) v(E) .
$$

Here, $\mu(\theta, E)$ is the wall-loss probability per collision, $\theta$ is the angle of incidence, and $\nu(E)$ is the frequency of wall collisions, which depends on the kinetic energy, $E$, of the neutrons and the geometrical shape of the storage volume.

For $W \ll V_{\mathrm{f}}$, which is valid for all materials useful for $\mathrm{UCN}$ guides and storage volumes, the reflection probability can be calculated from the reflected wave amplitude $R$ [37]:

$$
|R|^{2}=1-\mu(E, \theta)=1-2 \eta\left(\frac{E_{\perp}}{V_{\mathrm{f}}-E_{\perp}}\right)^{1 / 2} .
$$

In Eq. (4), $E_{\perp}$ is the kinetic energy from the velocity component in the direction perpendicular to the surface, $E_{\perp}=E \cos ^{2} \theta$. The average loss probability per wall collision for UCN of kinetic energy $E$ and integrated over all angles $\theta$ is then given by [37]

$$
\begin{aligned}
\bar{\mu}(E) & =2 \int_{0}^{1} \mu(E, \theta) \cos \theta d(\cos \theta) \\
& =2 \eta\left[\frac{V_{\mathrm{f}}}{E} \arcsin \left(\frac{E}{V_{\mathrm{f}}}\right)^{1 / 2}-\left(\frac{V_{\mathrm{f}}}{E}-1\right)^{1 / 2}\right] .
\end{aligned}
$$




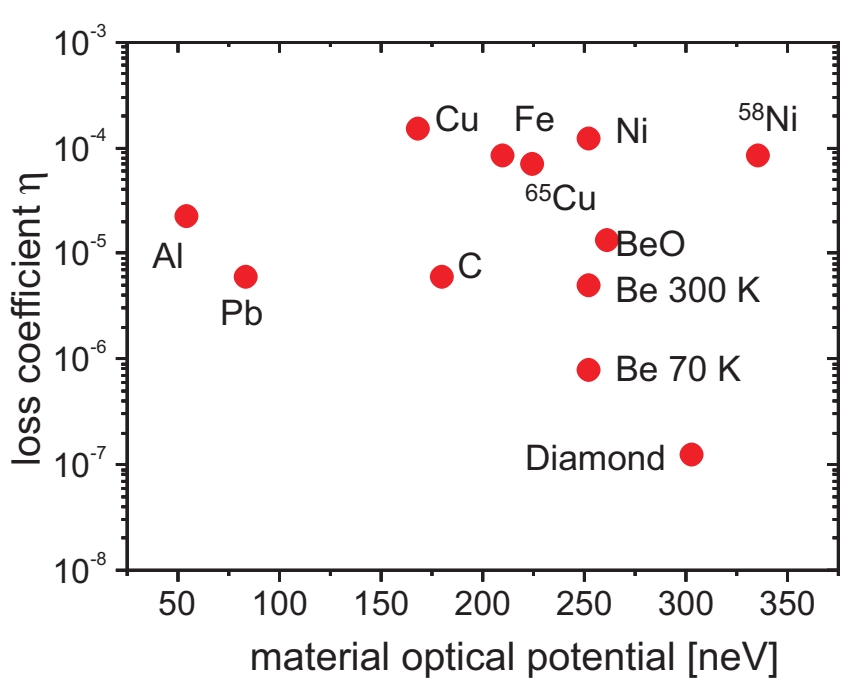

FIG. 1. Calculated loss coefficients $\eta$ vs the material optical potential $V_{\mathrm{f}}$ [Eq. (1)] of materials commonly used for storage of UCN (high potential, low losses) and UCN guides. The material optical potential of diamond-like carbon (DLC) is between that of diamond and that of carbon depending on the graphite/diamond ratio of the crystal structure. The data have been calculated from Eq. (1); see also Refs. [37,41].

The loss coefficients $\eta$ of commonly used materials for UCN storage and UCN guides calculated from Eq. (2) are plotted in Fig. 1; see also Refs. [37,41].

Experimentally, a variety of measurements has been performed to determine the losses during UCN storage. As a result, all experiments found higher losses of neutrons than expected from Eq. (2). A compilation of these experiments including searches for the cause of these anomalously large losses is given in Refs. [37,41] and references therein; for further reading, see Refs. [50-53]. The only conclusion that could be derived from the earlier experiments was that as far as UCN losses were concerned, all material surfaces seemed more or less identical and to possess some common feature that is responsible for the high losses. The problem of slits in the experimental setup being the reason for large losses was avoided in our experiment by the use of a magnetic valve; see Sec. III below.

The highest optical potential reported in literature [37] was for ${ }^{58} \mathrm{Ni}, V_{\mathrm{f}}=335 \mathrm{neV}$. ${ }^{2}$ Since nickel is ferromagnetic, polarized neutrons will be depolarized rapidly. The way out is to use nickel molybdenum alloys [55-57] with an optical potential of about $315 \mathrm{neV}$ at weight percentages of $85 \%$ $\mathrm{Ni}$ and $15 \%$ Mo $\left({ }^{58} \mathrm{NiMo} 85 / 15\right)$. The choice of the wall material for UCN guides should be adapted to the maximal available neutron energy from the respective UCN source. At PSI, the maximum available energy of the UCN is about $250 \mathrm{neV}$. Therefore, in a first generation, we have used alloys

\footnotetext{
${ }^{2}$ Investigations have been started to measure and apply coatings of cubic boron nitride $\left(\mathrm{c}^{11} \mathrm{BN}\right)$, cubic boron carbide $\left(\mathrm{c}^{11} \mathrm{~B}_{4} \mathrm{C}\right)$, and cubic carbon nitride $\left(\mathrm{cC}_{3} \mathrm{~N}_{4}\right)$ with expected optical potentials between 235 and $380 \mathrm{neV}$ [54].
}

of natural nickel and molybdenum with an optical potential around $220 \mathrm{neV}$ for our UCN guides, thereby avoiding the high cost of the pure isotope ${ }^{58} \mathrm{Ni}$.

In the nEDM experiment at PSI [7], the side walls of the neutron spin-precession chamber must be coated with a high-resistivity, nonmagnetic material of high optical potential. In principle, $\mathrm{BeO}$ fulfills these properties best. The drawbacks, however, are (i) its depolarization of the mercury comagnetometer vapor [58], (ii) its high toxicity, and (iii) the lack of available coating facilities.

Other candidate coatings are deuterated polystyrene (dPS) and deuterated polyethylene (dPE). Their optical potentials, $V_{\mathrm{f}}$, and other material parameters were determined [59]: $V_{\mathrm{f}}$ $(\mathrm{dPS})=(161 \pm 10) \mathrm{neV}$ and $V_{\mathrm{f}}(\mathrm{dPE})=(214 \pm 10) \mathrm{neV}$. The UCN losses at room temperature for $\mathrm{dPS}, \eta=(3 \pm 1) \times 10^{-4}$ [59], were similar to those of commonly used materials for UCN storage, e.g., diamond-like carbon (DLC) or beryllium [48] with optical potentials around $250 \mathrm{neV}[46,48,49,60$ 63] and low losses [52,64-67]. In the nEDM experiment at PSI [7], the high-voltage electrodes of the present chamber are coated with DLC while the walls are coated with dPS, which has the limiting optical potential $V_{\mathrm{f}}$ of the apparatus. Coating with $\mathrm{dPE}$ would further enhance the storable UCN density. Its optical potential $V_{\mathrm{f}}=(214.8 \pm 5.2) \mathrm{neV}[59,68]$ and the loss parameter $\eta=(1.3 \pm 0.3) \times 10^{-4}$ were recently measured [68]. The coating techniques for $\mathrm{dPE}$ are still under development $[68,69]$.

Depolarization of UCN was described in Refs. [48,49,7072]. It underlines that UCN scattering from surfaces is not completely coherent. The explanation of depolarization is so far hypothetical; it might originate from, e.g., impurities on the surfaces.

\section{EXPERIMENTAL SETUP}

Our experimental apparatus for the measurement of losses and depolarization probabilities is shown in Fig. 2 and described in detail in Ref. [73]. The first experimental results on diamond-like carbon and beryllium were published earlier $[48,49]$. The experimental apparatus consists of cylindrical sample tubes $1.5 \mathrm{~m}$ long, which are placed in a bore through the center of the iron yokes and poles of an H-type split-coil magnet. For the detection of the neutrons, we used the process

$$
n+{ }^{3} \mathrm{He} \rightarrow p+{ }^{3} \mathrm{H}
$$

in a gas mixture of $18 \mathrm{hPa}^{3} \mathrm{He}, 12 \mathrm{hPa} \mathrm{CO}$, and $1070 \mathrm{hPa} \mathrm{Ar}$.

The cylindrical sample tubes have an outer diameter of $80 \mathrm{~mm}$ and an inner diameter of $70 \mathrm{~mm}$ coated with the materials under investigation. The upper $90 \mathrm{~cm}$ of the tube serves as a storage volume which can be closed by a rapidly $(\sim 2 \mathrm{~s})$ switchable magnetic field of $1.52 \mathrm{~T}$ in the air gap (17, Fig. 2) of the H-type split-coil magnet (7, 8, Fig. 2). The midplane of this air gap defines our coordinate system with the height $z$ in the cylindrical tube and the radius $r$, with $r=z=0$ in the center of the bore.

In the magnetic field, the magnetic moments of the UCN are orientated parallel or antiparallel to the direction of the magnetic field and only one spin component of the originally unpolarized UCN can be stored above the air gap of the 


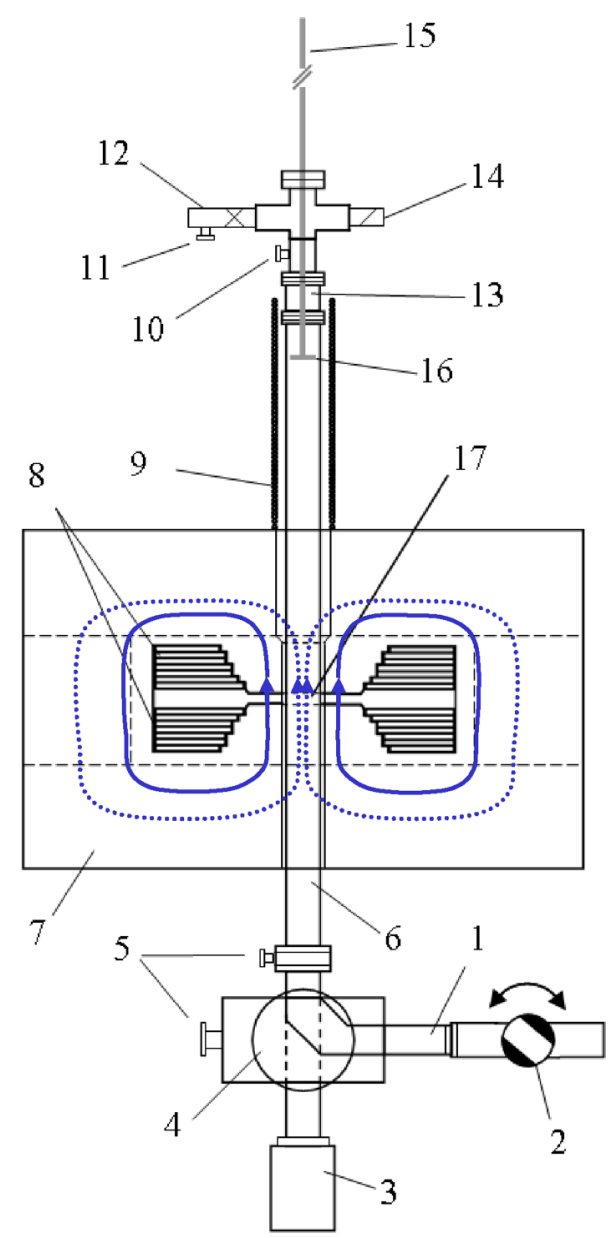

FIG. 2. Experimental apparatus for the investigation of materials for the storage of ultracold neutrons: 1 , neutron guide guide from the UCN source; 2, beam-line shutter; 3, UCN detector; 4, UCN switch between UCN guide from source to sample and sample to UCN detector; 5 , vacuum pumping ports; 6 , vertical UCN guide (sample) below magnetic field region; 7, magnet yoke; 8, magnet coils; 9, holding field coils; 10 , vacuum port to prepump; 11 , vacuum port to prepump; 12, vacuum pump (turbo pump); 13, extension vacuum tube; 14, vacuum sensor; $15, \mathrm{UCN}$ absorber with variable height; 16 , vertical UCN guide (sample) above magnetic field region (storage volume); and 17, magnetic field lines at $I=300 \mathrm{~A}$ in the air gap of the split-coil magnet. Solid lines: $B=2.7 \mathrm{~T}$ in the $3-\mathrm{cm}$ air gap. Dotted lines: $B=1.52 \mathrm{~T}$ in the center of the bore for the vertical UCN guide $(z=0, r=0)$.

magnet. ${ }^{3}$ Since these neutrons are pushed out of the magnetic field to lower field regions, they are termed low-field seekers (LFS).

There are quite some UCN present in the region of the strong magnetic field in the gap of the magnet while the field is ramped rapidly. Those with their magnetic moments parallel to the magnetic field are decelerated and trapped in the magnetic

\footnotetext{
${ }^{3}$ Storage of very slow neutrons in magnetic fields was first discussed by Vladimirski [74]. The first experiments were performed by Abov et al. [75].
}

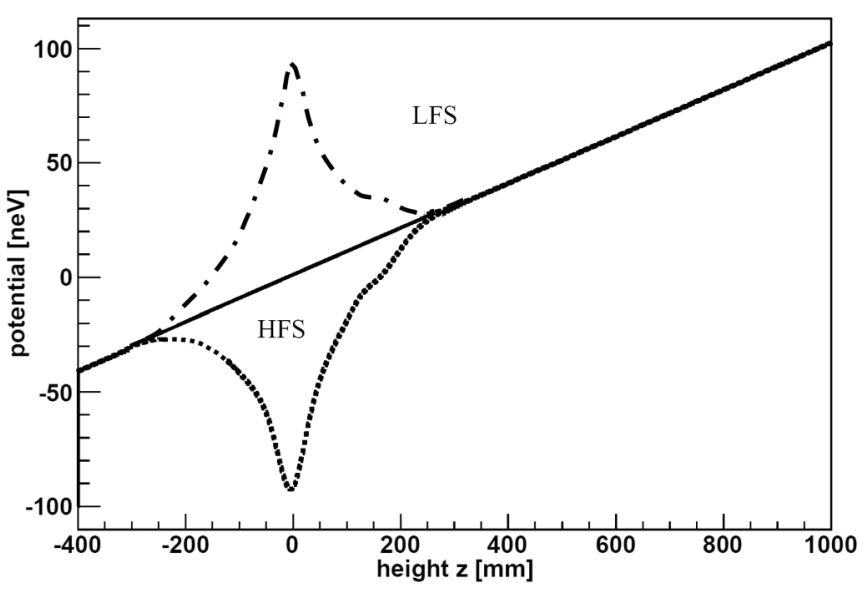

FIG. 3. Potential energy calculated from the known distribution of the magnetic field in the storage volume during UCN storage as a function of height. At small heights, the potential is mainly determined from the magnetic field in the air gap of the magnet. The slight kink around $z=150 \mathrm{~mm}$ originates from saturation effects in the iron where the bore diameter increases from 81 to $121 \mathrm{~mm}$. The gravitational potential energy rises linearly with height and dominates at larger $z$ values.

field region if their kinetic energy is on average $\sim 17 \mathrm{neV}$ immediately after the magnetic field is switched on. They are termed high-field seekers (HFS). Some of these high-fieldseeking UCN may, however, have energies up to $\sim 100 \mathrm{neV}$. This phenomenon of trapping UCN with two different spin directions in the same apparatus was published earlier [76].

We show in Fig. 3 the distribution of the potential energy in the apparatus as a function of height. The regions are indicated where two different spin states, HFS and low-field seekers (LFS), can be trapped. The calculation of the potential energy distribution is based on the measurements of the magnetic field along the vertical axis of the apparatus and two-dimensional calculations of the rotation-symmetric field distribution using the code POISSON SUPERFISH [77].

At the upper end, UCN are confined in the storage volume by gravity: The magnetic field of $B=1.52 \mathrm{~T}$ allows us to store UCN with energies corresponding to the potential energy of the magnetic field of $E_{\mathrm{B}}=\left|1.91 \mu_{\mathrm{N}} B\right| \approx 90 \mathrm{neV}$. Here, $\mu_{\mathrm{N}}$ is the nuclear magneton. This energy, in turn, corresponds to a maximum reachable height $h$ of the UCN in the Earth's gravitational potential $U_{\mathrm{g}}$ of $h=92 \mathrm{~cm}$ with $U_{\mathrm{g}} / h=102 \mathrm{neV} / \mathrm{m}$. Ultracold neutrons with higher energies are absorbed by a metal plate, coated with gadolinium, at the upper end of the storage volume (15, Fig. 2) or can pass the magnetic-field barrier and leave the storage volume at its lower end. The UCN are confined radially by the optical potential of the inner surface of the cylindrical sample tube. This principle of storage avoids mechanical gaps in the surface of the storage volume which are difficult to control, a serious cause of UCN loss, and hence would lead to a lower storagetime constant, mimicking higher losses. The measurements of storage-time constants with this apparatus allows for extraction of UCN loss coefficients of material walls and, simultaneously, 
the determination of the depolarization probability per wall collision, as presented below.

\section{Preparation of samples}

For most of the metallic coatings, the materials under investigation were sputtered directly onto the inner side of glass tubes. These tubes were produced by Schott AG, Mainz, Germany.

In order to remove contaminations from the glass surface, the tubes were cleaned in a Mucasol [78] bath at a temperature of $\sim 50^{\circ} \mathrm{C}$ and rinsed with demineralized water. After this wet cleaning, the tubes were heated in vacuum for $12 \mathrm{~h}$ to about $150^{\circ} \mathrm{C}$. Still hot, the tubes were transferred to the sputter chamber, which was pumped to a vacuum of $10^{-7}$ mbar. The glass tubes then were coated at their inner surface with a layer typically $500 \mathrm{~nm}$ thick. For one of the NiMo 85/15 samples, we used a tube of aluminium with roughness $\sim 250 \mathrm{~nm}$ and the same dimensions as the glass tubes.

The deuterated polystyrene (dPS) sample was produced by drilling holes of $70 \mathrm{~mm}$ diameter into three 50 -cm-long polystyrene rods of outer diameter $80 \mathrm{~mm}$. These three tubes were cleaned and glued together at steplike formed borders to form a tube $1.5 \mathrm{~m}$ long as the glass tubes. Removable rings were fixed at the two ends with an inner opening of $\sim 50 \mathrm{~mm}$. Next, $1.1 \mathrm{~g}$ of deuterated polystyrene $(98 \%)$ was dissolved in $150 \mathrm{~cm}^{3}$ of toluene and filled into the tube placed horizontally on a rotatable gadget under an exhaust hood. The tube rotated with a frequency of $0.02 \mathrm{~Hz}$ for $24 \mathrm{~h}$ until the toluene had completely evaporated. After four days of drying, the process was repeated with $1.2 \mathrm{~g}$ of deuterated polystyrene $(98 \%)$ and $150 \mathrm{~cm}^{3}$ of deuterated $(99.5 \%)$ toluene. The final layer of deuterated polystyrene inside the tube was $\sim 5 \mu \mathrm{m}$.

\section{DATA COLLECTION}

The experiment was performed at the beam line PF2/EDM $[79,80]$ of the Institut Laue-Langevin (ILL) in Grenoble, France, and at the UCN source at the Paul Scherrer Institute in Villigen, Switzerland [15-18]. The vacuum in the sample tubes was typically $1 \times 10^{-6}$ mbar. Ultracold neutrons from the UCN turbine [81] or the PSI-UCN-West beam entered the experimental apparatus through an UCN guide (1, Fig. 2) and a shutter (2, Fig. 2) in the open position. With the UCN switch (4, Fig. 2) in filling position, UCN are deflected into the vertically mounted sample tube (6 and 16, Fig. 2).

After $20 \mathrm{~s}$ of filling, the maximum neutron density in the storage volume of the sample (the upper $90 \mathrm{~cm}$ ) was reached and the magnetic field was switched on to close the UCN volume. The field was raised within $\sim 2 \mathrm{~s}$ to $90 \%$ of the maximum electric current, corresponding to $1.45 \mathrm{~T}$. Then, the mechanical neutron switch (4, Fig. 2) was moved from filling position to emptying position, thereby blocking the UCN from the source. The beam-line shutter (2, Fig. 2) was closed for improved background suppression.

After switching on the magnetic field, we waited for $100 \mathrm{~s}$. In a previous work $[49,82]$, it was found by Monte Carlo simulations using GEANT4UCN $[82,83]$ that during these $100 \mathrm{~s}$ all HFS in the storage volume above the magnetic field region and all LFS with total energies higher than $\sim 90 \mathrm{neV}$ should have either been absorbed at the top of the storage volume or have passed the magnetic field and fallen into the neutron counter (3, Fig. 2), where they were detected. Later in the analysis, it was, however, found (see Sec. V D below) that the 100-s waiting time was too short to sort out all UCN of higher energies. In the following, we term the time to sort out the UCN of higher energies "cleaning time" or "spectral cleaning." Furthermore, it was found that the cleaning time depended on absorber position: Between $70 \mathrm{~cm}$ and $90 \mathrm{~cm}$, the cleaning time would have been $220 \mathrm{~s}$, and between $40 \mathrm{~cm}$ and $60 \mathrm{~cm}$, it would have been $170 \mathrm{~s}$. For absorber positions of $30 \mathrm{~cm}$ and below and for the ferromagnetic samples, the cleaning time of $100 \mathrm{~s}$ was sufficient. These extended cleaning times were taken into account in the analysis.

During data taking, the magnetic field was raised after $100 \mathrm{~s}$ to $100 \%$, i.e., $1.52 \mathrm{~T}$; and the remaining $\mathrm{UCN}$ were stored for times reaching up to $370 \mathrm{~s}$. Neutrons which were depolarized during such holding times, e.g., by an interaction with the nuclei of the surface of the sample during a wall collision, passed the magnetic-field barrier and were registered in the neutron detector. After the selected holding time, the magnetic field was rapidly switched off $(t<2 \mathrm{~s})$ and the remaining UCN were detected during an emptying time of 35 s. Figure 4 shows the detector counts over time including filling (detector closed), spectral cleaning, storage, and emptying. Here, the absorber position was $90 \mathrm{~cm}$ and the overall cleaning time was $220 \mathrm{~s}$. For absorber position of $50 \mathrm{~cm}$, the corresponding detector counts are shown in Fig. 5; Fig. 6 shows a similar scheme for the HFS.

\section{DATA ANALYSIS}

With the sequences shown in Figs. 4, 5, and 6, the wall losses and depolarization probabilities of UCN interacting with the surface material of the sample tube can be determined simultaneously. The storage-time constant of the storage volume under investigation, $\tau_{\mathrm{st}}$, is determined from the UCN counts, $N_{\mathrm{i}}$, after holding times $t_{\mathrm{i}}$. It depends on (i) the lifetime of the free neutron, $\tau_{\mathrm{n}}$, (ii) the time constant for the wall losses, $\tau_{\mu}$, which is the inverse of the average loss rate, cf. Eq. (3), and (iii) the time constant $\tau_{\beta}$ originating from losses through spin flips during storage, $1 / \tau_{\beta}=\beta \nu$, where $\beta$ is the depolarization probability per wall collision. In total, we get

$$
\frac{1}{\tau_{\mathrm{st}}}=\frac{1}{\tau_{\mathrm{n}}}+\frac{1}{\tau_{\mu}}+\frac{1}{\tau_{\beta}}=\frac{\ln \left(N_{\mathrm{s}} / N_{\mathrm{i}}\right)}{t_{\mathrm{i}}-t_{\mathrm{s}}}
$$

Here, $N_{\mathrm{s}}$ is the number of neutrons at the beginning of storage $t_{\mathrm{s}}$ (cf. Sec. V D below); $N_{\mathrm{i}}$ is the remaining number of neutrons counted after a holding time, $t_{\mathrm{i}}$. Upscattering of UCN from residual gases as described in Ref. [84] can be neglected at our vacuum conditions $\left(1 \times 10^{-6}\right.$ mbar or better $)$ and our statistical uncertainties for $\tau_{\text {st }}$; see below.

The number of spin-flipped neutrons is

$$
\frac{d N_{\mathrm{sp}}}{d t}=N(t) v \beta
$$

Here, $N(t)$ is the number of stored neutrons at time $t$. By integrating Eq. (8) between time $t_{\mathrm{s}}$ and $t_{\mathrm{i}}$ and assuming one 


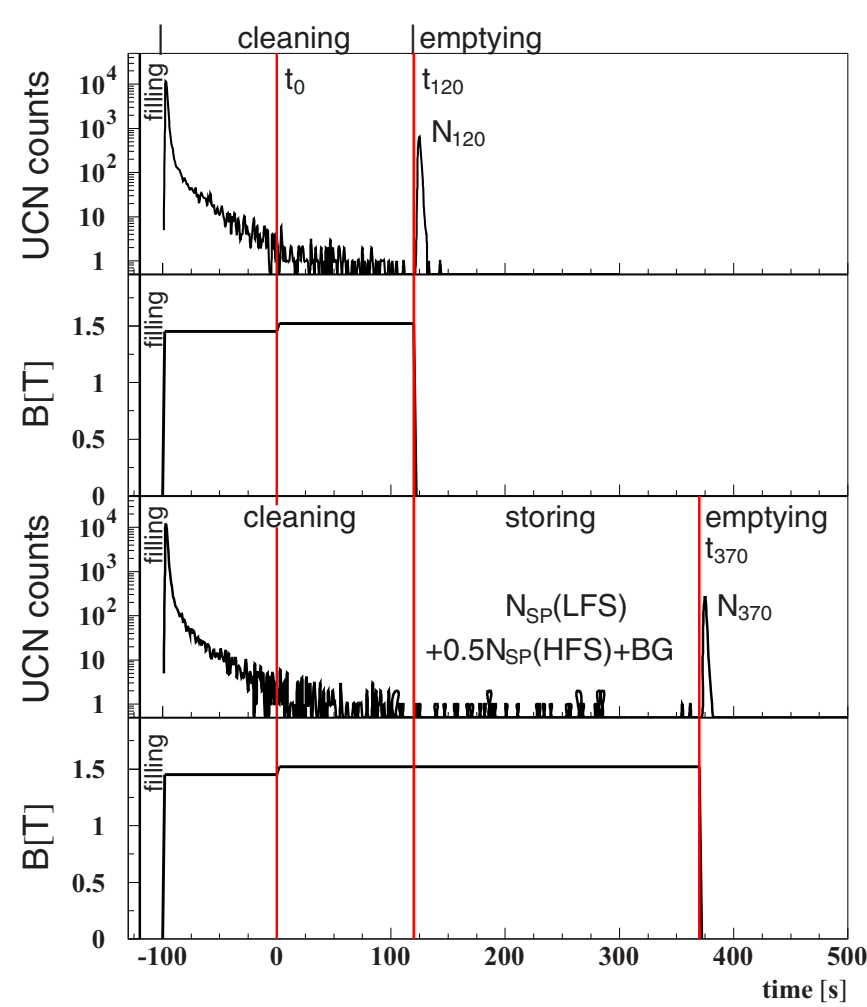

FIG. 4. Detector counts for the sequence filling ( -120 to $-100 \mathrm{~s})$, cleaning $(-100$ to $+120 \mathrm{~s})$, storing, and emptying for storing times $t=0 \mathrm{~s}$ (top) and $t_{370}-t_{120}=250 \mathrm{~s}$ (bottom); $N_{120}$, $N_{370}$, and $N_{\text {sp }}$ are the neutron counts at $t_{120}, t_{370}$, and the spin-flipped neutrons, respectively. The data are from the NiMo 88/12 sample with the absorber at $90 \mathrm{~cm}$. The events during storage are attributed to (i) spin-flip events of the LFS, (ii) approximately half of the spin-flipped events of the HFS, namely those which are emitted downward toward the detector; the other half is accelerated upward into the LFS region where they are stored, and (iii) background (BG). As mentioned in the text, the cleaning time depends on the absorber position. The magnetic field $\mathrm{B}$ at $z=0, r=0$ as a function of time is also shown. It is ramped from $90 \%$ of the current to $100 \%$ at $t=0$.

single exponential, one obtains

$$
\begin{aligned}
N_{\mathrm{sp}}(t) & =v \beta \int_{t_{\mathrm{s}}}^{t_{\mathrm{i}}} N(t) d t \\
& =v \beta \int_{t_{\mathrm{s}}}^{t_{\mathrm{i}}} N_{\mathrm{s}} e^{-t / \tau_{\mathrm{st}}} d t \\
& =\tau_{\mathrm{st}} \beta v\left(N_{\mathrm{s}}-N_{\mathrm{s}} e^{-t_{\mathrm{i}} / \tau_{\mathrm{st}}}\right) \\
& =\tau_{\mathrm{st}} \beta v\left(N_{\mathrm{s}}-N_{\mathrm{i}}\right) .
\end{aligned}
$$

Here, $N_{\mathrm{sp}}(t)$ are the UCN counted during storage, representing the spin-flipped and escaped UCN. With this, the time constant, $\tau_{\beta}$, of the spin flips can be determined directly from the spinflip counts, $N_{\text {sp }}$; see Figs. 4-6. For one energy, we get

$$
\frac{1}{\tau_{\beta}}=\beta \nu(E)=\frac{N_{\mathrm{sp}}\left(t_{\mathrm{i}}\right)}{N_{\mathrm{s}}-N_{\mathrm{i}}} \frac{1}{\tau_{\mathrm{st}}} .
$$

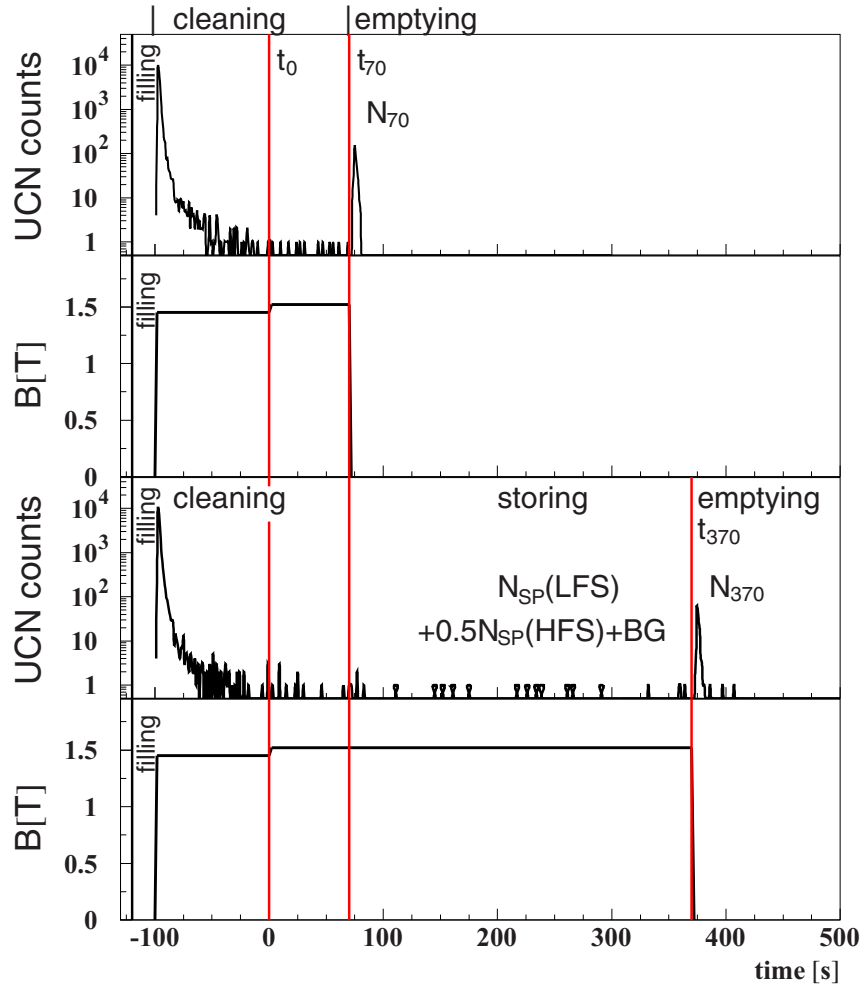

FIG. 5. Same as Fig. 4. The absorber position is $50 \mathrm{~cm}$ and the cleaning time is $170 \mathrm{~s}$.

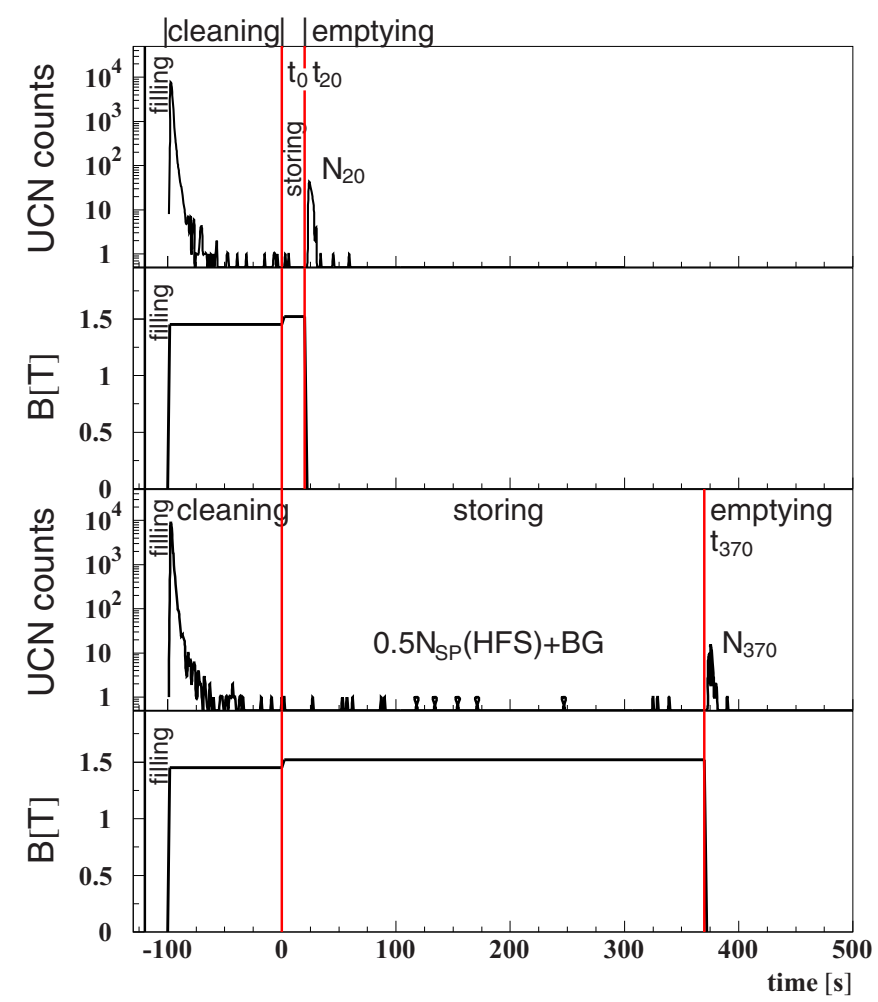

FIG. 6. Same as Fig. 4. Here, the absorber position is $30 \mathrm{~cm}$ and the cleaning time $100 \mathrm{~s}$. 


\section{A. UCN energy distribution}

In our experiment, we have an energy distribution of UCN ranging from $0 \mathrm{neV}$ to a total energy, $E_{0}(z)=V(z)+E_{\mathrm{kin}}$, of $90 \mathrm{neV}$, with the latter depending on the position of the absorber. The maximum kinetic energy depends again on the position of the absorber and ranges from 0 to $60 \mathrm{neV}$. Because of their different velocities, UCN with different kinetic energies have different frequencies of wall collisions. Thus, $v(E)$ in Eq. (10) has to be substituted by

$$
\langle v\rangle=\frac{\sum_{\mathrm{i}} v_{\mathrm{i}}\left(E_{0}\right) g_{\mathrm{i}}\left(E_{0}\right)}{\sum_{\mathrm{i}} g_{\mathrm{i}}\left(E_{0}\right)},
$$

with $g_{\mathrm{i}}\left(E_{0}\right)$ from the differential energy distribution of the UCN; see below.

\section{Energy distribution of the LFS}

As was shown before [76], we have for absorber positions $z>30 \mathrm{~cm}$ both LFS and HFS stored in the apparatus. For the determination of the storage-time constants, $\tau_{\mathrm{st}}$, as well as the spin-flip counts, $N_{\text {sp }}$, of the LFS, the corresponding contributions from the HFS have to be evaluated and subtracted. Also for the determination of $\langle v\rangle$, we need to know the energy distribution of the stored UCN. We measured this by varying the height of the absorber (15, Fig. 2). The maximal storable total UCN energy is then defined by the absorber height. Figure 7 shows the UCN counts as a function of the height $z_{\mathrm{a}}$ of the UCN absorber. The sample is NiMo82/18 and the total cleaning time is $220 \mathrm{~s}$, i.e., from $t=-100 \mathrm{~s}$ to $t=+120 \mathrm{~s}$.

As mentioned above, the low-energy part of the spectrum follows a $v^{2}$ dependence and its integral is proportional to $v^{3}$ or $E_{\mathrm{kin}}^{3 / 2}$. In order to obtain a continuous distribution, we fitted the data to a function with two free parameters,

$$
N(z)=N_{120}(30 \mathrm{~cm})+P_{1}\left(z-P_{2}\right)^{3 / 2} .
$$

Here, $N_{120}(30 \mathrm{~cm})$ is the measured HFS counts at absorber position $30 \mathrm{~cm}, P_{1}$ is a normalization factor, and $P_{2}$ is the minimum of the potential energy $(z \approx 30 \mathrm{~cm})$. The fitted function in the case of NiMo82/18 is

$$
N(z)=49.1+2.47(z-33.4)^{3 / 2},
$$

with a $\chi^{2}$ of 1.19 for one degree of freedom corresponding to a confidence level of about $30 \%$. This fit [Eq. (12)] has been applied to the data points of all paramagnetic samples with similar results.

From the data (black dots), we derive a differential spectrum (see Fig. 7, open squares), and from Eq. (13), we obtain the derivative of $N(z)$ with respect to $z$,

$$
\frac{d N}{d z}=1.5 P_{1}\left(z-P_{2}\right)^{1 / 2},
$$

shown as a line through the open squares. This differential distribution, corresponding to the $g_{\mathrm{i}}\left(E_{0}\right)$ of Eq. (11) above, is needed for the evaluation of $\beta$ and $\eta$ values; see Secs. VI A and VI A below.

In Fig. 7, the UCN counts between $z_{\mathrm{a}} \approx 30 \mathrm{~cm}$ and $z_{\mathrm{a}} \approx$ $10 \mathrm{~cm}$ are constant within their uncertainties and amount to about $4.5 \%$ of the total counts measured at $z_{\mathrm{a}}=90 \mathrm{~cm}$; they

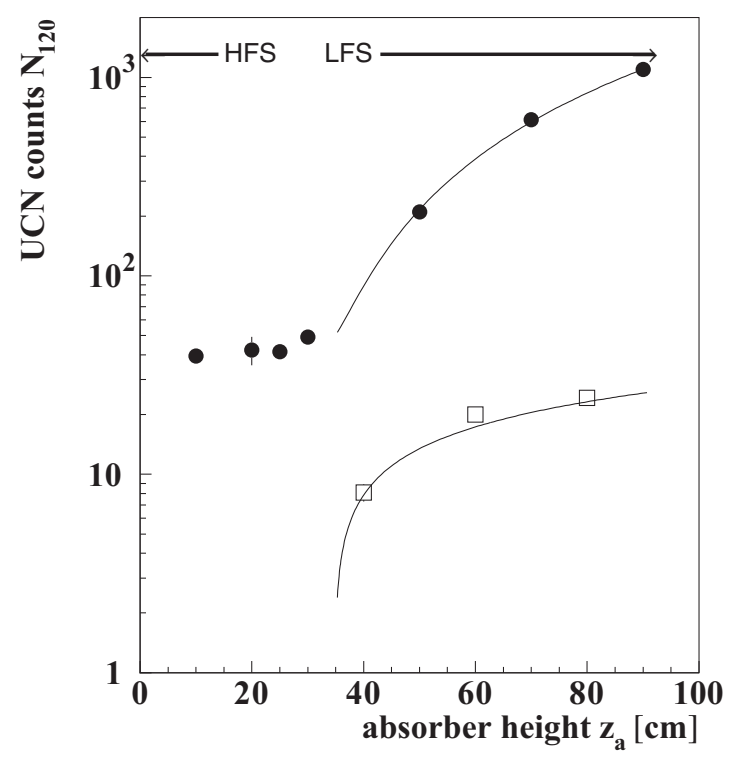

FIG. 7. Counts of UCN as a function of the absorber height $z_{\mathrm{a}}$ after $20 \mathrm{~s}$ of filling and $220 \mathrm{~s}$ of spectral cleaning. The sample is NiMo82/18. Black dots, measured integral UCN distribution; and open squares, differential UCN distribution. The differential distribution was obtained from the differences in count rate at $z(i)$ and $z(i-1)$, where $z(i-1)=z(i)-20 \mathrm{~cm}$. The integral distribution was fitted with a function $N_{120}(z)=N_{120}(30 \mathrm{~cm})+P_{1}\left(z-P_{2}\right)^{3 / 2}$ and is plotted as the line through the data points. Here, $P_{1}=2.47 \pm$ 0.09 is a normalization factor, $P_{2}=33.4 \pm 1.0$ is the minimum of the potential energy, and $N_{120}(30 \mathrm{~cm})=49.1$ is the measured number of HFS at $z_{\mathrm{a}}=30 \mathrm{~cm}$. The $\chi^{2}$ of the fit is 1.2 for one degree of freedom. The line through the experimental differential data is the derivative of $N_{120}$ with respect to $z, d N_{120} / d z=3.7(z-33.4)^{1 / 2}$. The agreement between the fitted value for $P_{2}$ and the calculated one from the magnetic field distribution $(23 \mathrm{~cm})$ is only fair but good enough for our purposes.

decrease to zero at $z_{\mathrm{a}}=0 \mathrm{~cm}$. The neutrons in the region of the high magnetic field below $z \approx 10 \mathrm{~cm}$ have opposite spins compared to the LFS and were identified as HFS trapped during the rapid ramping of the field [76].

\section{B. Determination of the wall collision frequency $v(E)$}

In order to determine the wall-loss coefficients and the probabilities of depolarization per wall collision, one needs to know the frequency $v(E)$ of collisions of the UCN at the sample walls; cf. Eqs. (3) $\left(\tau_{\mu}\right)$ and (10) $\left(\tau_{\beta}\right)$. This was determined by two different methods, (i) kinetic gas theory and (ii) Monte Carlo simulations.

\section{Kinetic gas theory}

If UCN are stored in vessels with material walls of small surface roughness (typical roughness amplitudes of a few nanometers), it was shown [37] that these UCN behave in many aspects like an ideal gas. The basic relation between the UCN flux $J$, their velocity $v$, and their density $n$ is

$$
J=\frac{1}{4} n v
$$


Low-field-seeking UCN moving along the vertical (z) axis of the experiment (cf. Fig. 2) are exposed to gravity and the strong magnetic field of the dipole magnet in the region $0 \leqslant z \leqslant 30 \mathrm{~cm}$. They experience a potential $V(z)$; see Fig. 3 . This potential has a minimum, $V_{\min }=29 \mathrm{neV}$ at $z \approx 230 \mathrm{~mm}$. Ultracold neutrons with a total energy of $E_{0}$ are confined to a limited region, $z_{\mathrm{b}}$ and $z_{\mathrm{t}}$, along the $z$ axis. Here, subscripts $\mathrm{b}$ and $\mathrm{t}$ stand for bottom and top, respectively. For the LFS, these two boundary values are determined by $V\left(z_{\mathrm{b}}\right)=E_{0}$ left of $V_{\min }$ at about $230 \mathrm{~mm}$ (see Fig. 3) and $V\left(z_{\mathrm{t}}\right)=E_{0}$ right of $V_{\min }$ in the gravitational part of the potential, respectively. The HFS can oscillate between about $-200 \mathrm{~mm}$ and $+100 \mathrm{~mm}$.

The UCN velocity at an arbitrary point $z$ is

$$
v\left(E_{0}, z\right)=\sqrt{\frac{2\left[E_{0}-V(z)\right]}{m}} .
$$

Following Ref. [37] at an arbitrary position $z$, the UCN density can be expressed by

$$
n\left(E_{0}, z\right)=\frac{N \sqrt{E_{0}-V(z)}}{\pi \rho^{2} \int_{z \mathrm{~b}}^{z_{\mathrm{b}}} \sqrt{E_{0}-V(z)} d z},
$$

with $N$ the number of $\mathrm{UCN}$ in the volume. The differential wall collision rate for one single $\mathrm{UCN}$ is

$$
\frac{d \nu}{d z}\left(E_{0}, z\right)=\frac{2 \pi \rho J\left(E_{0}, z\right)}{N},
$$

where $\rho$ is the inner radius of the sample tubes. Using Eqs. (15)-(17), one can transform Eq. (18) to

$$
\frac{d \nu}{d z}\left(E_{0}, z\right)=\frac{v\left(E_{0}, z\right)}{2 \rho} \frac{\sqrt{E_{0}-V(z)}}{\int_{z_{\mathrm{b}}}^{z_{\mathrm{t}}} \sqrt{E_{0}-V(z)} d z} .
$$

As an example, we show in Fig. 8 the differential frequency of wall collisions $d v / d z$ averaged over all energies $E_{0}<$ $90 \mathrm{neV}$. The differential frequency of wall collisions is

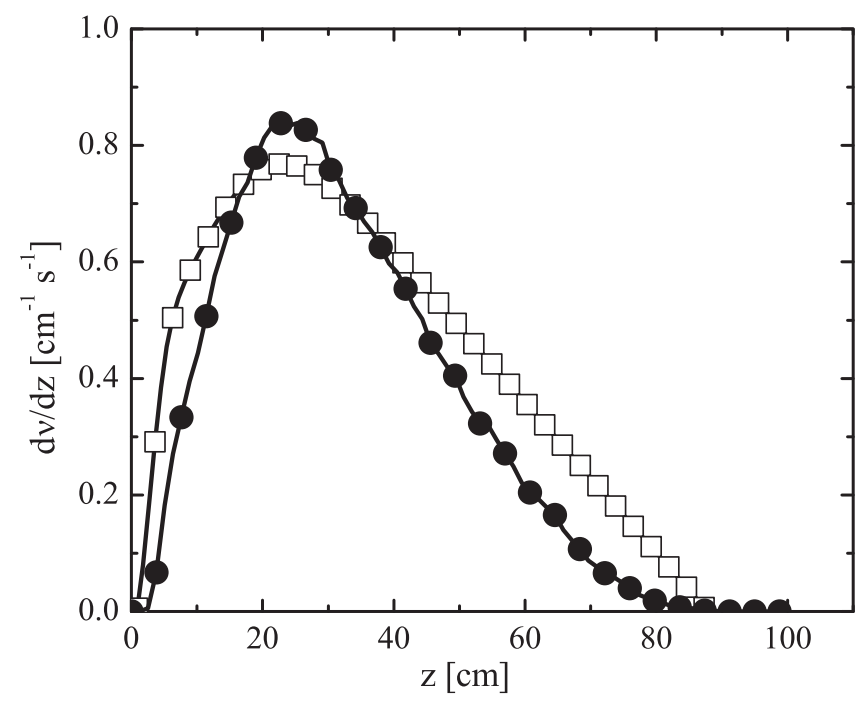

FIG. 8. Result for the differential frequency of wall collisions $d v / d z$ for LFS, obtained with a MC code (black line with black dots), and a comparison with a theoretical model, using the kinetic gas theory for UCN (black line with open squares). The absorber was set to $z_{\mathrm{a}}=90 \mathrm{~cm}$.

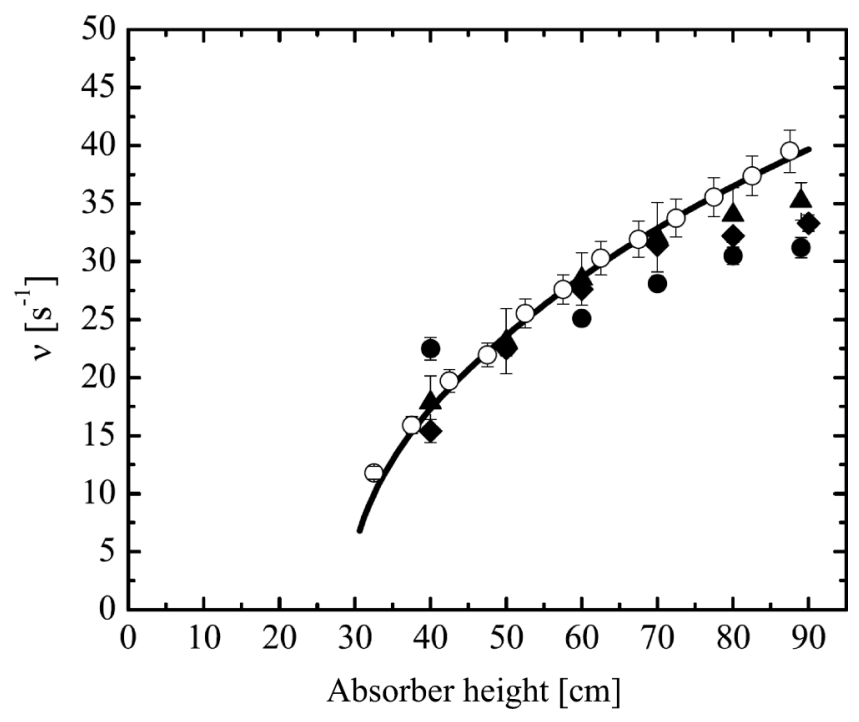

FIG. 9. Collision rates $v(z)$ of $\mathrm{UCN}$ as a function of the absorber height. The black line is calculated from kinetic gas theory. The Monte Carlo simulations are as follows: (i) open circles, Ref. [85]; (ii) black circles, Ref. [86]; (iii) diamonds, Ref. [87]; and (iv) black triangles, Ref. [88].

maximal at the minimum of the potential energy $V(z)$, as expected. Integration of Eq. (19) over the height $z$ leads to the averaged frequency of wall collision $v\left(E_{0}\right)$; see Fig. 9 . The total energy $E_{0}$ corresponds to the absorber height $z_{\mathrm{a}}$ via the relation $E_{0}=m_{\mathrm{n}} g z_{\mathrm{a}}\left(m_{\mathrm{n}}\right.$, neutron mass; $g$, gravitational constant). The maximal achievable kinetic energy of UCN in the sample tube is then $E_{\max }=E_{0}-V_{\min } \approx 60 \mathrm{neV}$ for $z_{\mathrm{a}}(\max )=90 \mathrm{~cm}$.

\section{Monte Carlo simulations}

We computed the collision rate of the UCN on the walls as a function of their total energy with a Monte Carlo code develloped at PSI, dedicated to and optimised for this experiment. The input for this code is the measured magnetic-field map in cylindrical coordinates with a high spatial resolution. We used bilinear interpolation in order to calculate the field gradients in radial and vertical directions at every point. These gradients were converted into Cartesian coordinates. Thus, at every point within the vertical guide, the magnetic and gravitational accelerations were known. With the Runge-Kutta-4 method, we calculated the trajectories of our sampling UCN starting with random initial conditions (initial energy relative to $z=0$, isotropic angular distribution), fixing the initial height corresponding to the minimum of the potential energy.

After storing the UCN for $100 \mathrm{~s}$, we sorted the number of collisions during $20 \mathrm{~s}$ into histograms as a function of their total energy relative to $z=0$, and thus calculated the collision rate averaged over time and height for every energy bin. By normalization with the number of UCN in each energy bin, we obtained the collision rate per UCN as a function of their total energy, i.e., as a function of $z$; see Table II and Fig. 9. We see remarkably good agreement with the analytic calculation based on kinetic gas theory; see Sec. V B 1 above. In order to check the precision of the MC simulation of the 
TABLE II. Collision rate of the low-field seeker UCN as a function of the absorber height. For the high-field seekers (absorber heights less than $30 \mathrm{~cm})$, the collision rate is $(65 \pm 2) \mathrm{s}^{-1}$.

\begin{tabular}{cc}
\hline \hline $\begin{array}{c}\text { Absorber height } \\
{[\mathrm{cm}]}\end{array}$ & $\begin{array}{c}\text { Collision rate } v \pm \Delta v \\
{\left[\mathrm{~s}^{-1} \mathrm{UCN}^{-1}\right]}\end{array}$ \\
\hline 32.5 & $11.8 \pm 0.6$ \\
37.5 & $15.9 \pm 0.8$ \\
40.0 & $17.8 \pm 1.0$ \\
42.5 & $19.7 \pm 1.0$ \\
47.5 & $21.9 \pm 1.0$ \\
50.0 & $23.7 \pm 1.1$ \\
52.5 & $25.5 \pm 1.2$ \\
57.5 & $27.6 \pm 1.3$ \\
60.0 & $29.0 \pm 1.4$ \\
62.5 & $30.3 \pm 1.5$ \\
67.5 & $31.9 \pm 1.6$ \\
70.0 & $32.8 \pm 1.6$ \\
72.5 & $33.8 \pm 1.6$ \\
77.5 & $35.6 \pm 1.7$ \\
80.0 & $36.5 \pm 1.7$ \\
82.5 & $37.4 \pm 1.7$ \\
87.5 & $39.5 \pm 1.8$ \\
90.0 & $40.4 \pm 1.8$ \\
\hline
\end{tabular}

trajectory in the high magnetic field, we also computed the deviation between the final potential energy from the field map after $120 \mathrm{~s}$ of storage and the potential energy obtained from the corresponding final kinetic energy. The accuracy in the potential energy was $0.003 \mathrm{neV}$ after $120 \mathrm{~s}$ of storage and the precision (standard deviation) was $0.2 \mathrm{neV}$. For this precision, the necessary step size in the Runge-Kutta-4 method was $100 \mu \mathrm{m}$.

In total, four independent MC codes were developed at PSI [85], TUM [86,87], and the university in Mainz [88]. Their results agree fairly well with each other; see Fig. 9.

For the determination of the averaged collision rate of the HFS in the Monte Carlo model as described above, we used a vertical cylinder with inner diameter of $70 \mathrm{~mm}$ coated with a material of optical potential $V_{\mathrm{f}}$; see Table I. We assume a coefficient for diffuse reflection of 0.02 and a loss parameter of $2 \times 10^{-4}$. A more precise input for these parameters does not change the result significantly.

In the simulation, the magnetic field can be switched on and off. We fill the volume from $z=-75 \mathrm{~cm}$ with UCN of an energy distribution $d N / d E \sim E^{0.5}$. Then, we switch on the magnetic field and store the UCN for several tens of seconds and calculate the collision rate. Our result, averaged over all possible kinetic energies of the HFS is

$$
\begin{aligned}
& \langle v\rangle(\mathrm{HFS})=(65 \pm 2) \mathrm{Hz} . \\
& \text { C. Data and background }
\end{aligned}
$$

An example of the raw data from our sample NiMo88/12 is shown in Fig. 10 for different absorber positions. As can be seen from Fig. 7, the data at absorber position $30 \mathrm{~cm}$ are solely high-field-seeking UCN. This can also be seen from Fig. 11, where the data at absorber positions $30 \mathrm{~cm}$ and 20 $\mathrm{cm}$ are shown.

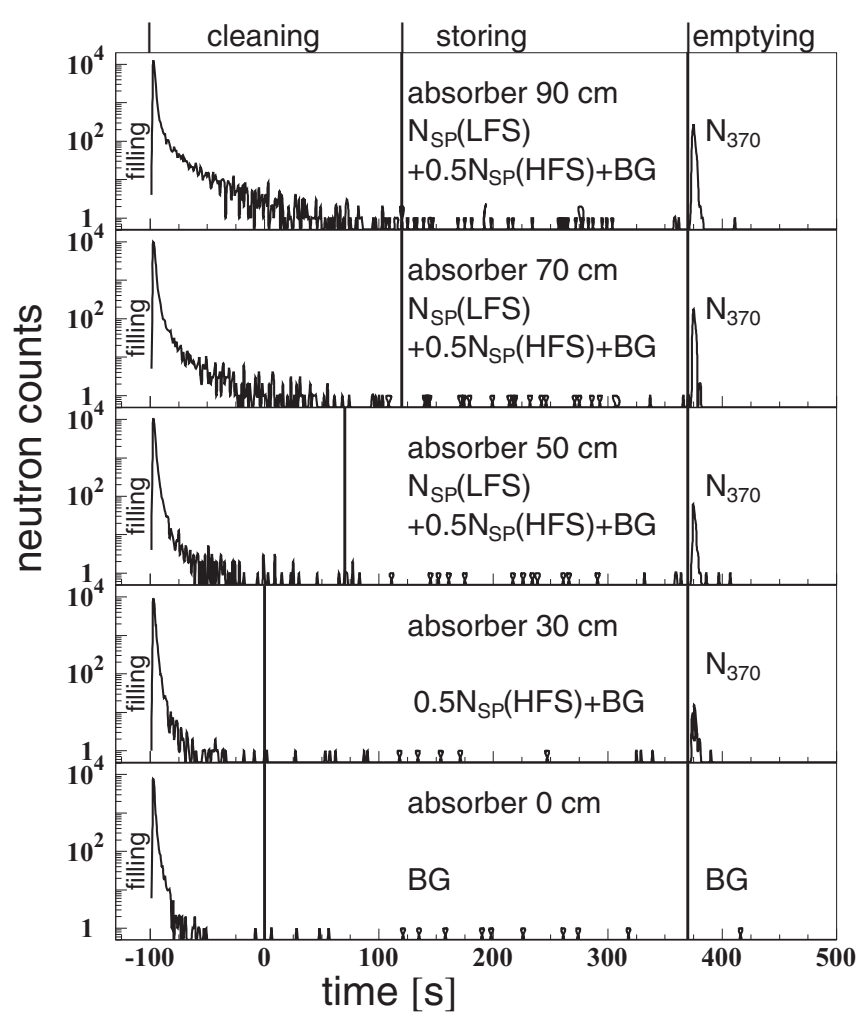

FIG. 10. Detector counts during data taking at different absorber positions: $90,70,50,30$, and $0 \mathrm{~cm}$. The sample is NiMo88/12.

For the presentation of our results, see Sec. VI below, we calculate the depolarization probabilities per wall collision, $\beta$, and the wall loss parameter, $\eta$. Both parameters are inversely proportional to the wall collision frequencies; see Eqs. (30), (31), (35), and (36) below. Since the HFS have a higher wall collision frequency than the LFS, see Eq. (20), their numbers of depolarization and loss events which are proportional to the number of wall collisions represent an additional background for the analysis of the low-field-seeking UCN. Besides HFS, the data at absorber position $30 \mathrm{~cm}$ (or $20 \mathrm{~cm}$ ) contain also background events common to all absorber heights. That is, for

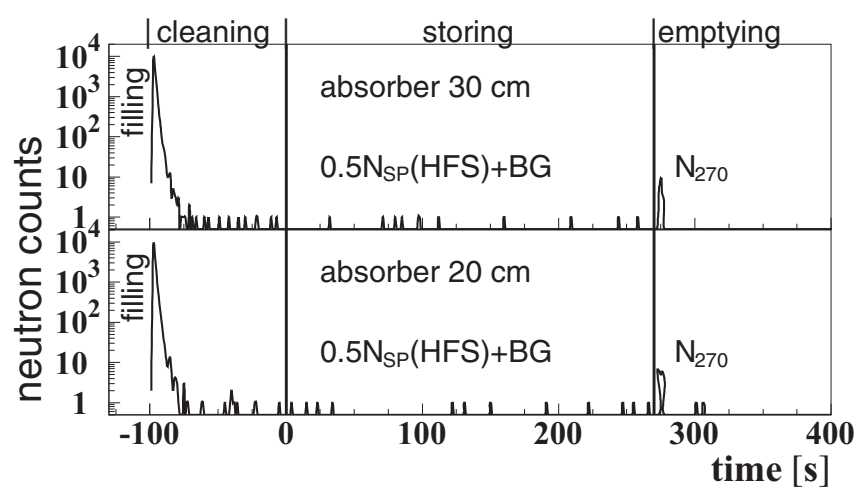

FIG. 11. Neutron spectra at absorber positions 30 and $20 \mathrm{~cm}$. The sample was NiMo $82 / 18$ and the holding time is $270 \mathrm{~s}$. The counts for $N_{\text {sp }}$ and $N_{270}$ averaged over all runs are $15.6 \pm 0.9$ and $21.1 \pm 1.1$ for $z=30 \mathrm{~cm}$ and $13.0 \pm 2.5$ and $19.5 \pm 3.1$ for $z=20 \mathrm{~cm}$. 
the analysis of the LFS, the data at 30-cm absorber positions have to be subtracted from the data taken at higher absorber positions; see Fig. 10:

(1) $N_{\mathrm{i}}$, i.e., the HFS counts in the emptying peak and

(2) $0.5 N_{\mathrm{sp}}(\mathrm{HFS})+B G$, half of the spin flipped HFS plus background (BG).

This means, e.g., for the emptying peak of the LFS at absorber position $90 \mathrm{~cm}$

$$
N_{\mathrm{i}}(\text { LFS })=N_{\mathrm{i}}(90 \mathrm{~cm})-N_{\mathrm{i}}(30 \mathrm{~cm}),
$$

where

$$
N_{\mathrm{i}}(90 \mathrm{~cm})=\sum_{t_{\mathrm{i}}}^{t_{\mathrm{i}+35}} N(t)(90 \mathrm{~cm}) .
$$

with $N(t)(90 \mathrm{~cm})$ being the data at absorber position $z_{\mathrm{a}}=$ $90 \mathrm{~cm}$ and an emptying time of $35 \mathrm{~s}$, and

$$
N_{\mathrm{i}}(30 \mathrm{~cm})=\sum_{t_{\mathrm{i}}}^{t_{i+35}} N(t)(30 \mathrm{~cm})
$$

with $N(t)(30 \mathrm{~cm})$ being the data at absorber position $z_{\mathrm{a}}=$ $30 \mathrm{~cm}$.

For the spin-flipped LFS we used

$$
N_{\mathrm{sp}}(\mathrm{LFS})=\sum_{t_{\mathrm{s}}}^{t_{\mathrm{i}}} N(t)(90 \mathrm{~cm})-\sum_{t_{\mathrm{s}}}^{t_{\mathrm{i}}} N(t)(30 \mathrm{~cm}),
$$

where $t_{\mathrm{s}}$ is the beginning of storing, i.e., $t_{\mathrm{s}}=120 \mathrm{~s}$ for the position of the absorber at 90 and $70 \mathrm{~cm}, t_{\mathrm{s}}=70 \mathrm{~s}$ for $50 \mathrm{~cm}$, and $t_{\mathrm{s}}=0 \mathrm{~s}$ for the HFS and the ferromagnetic samples.

In the analysis of the HFS, i.e., for absorber positions of $30 \mathrm{~cm}$ or less, a background has to be subtracted:

$$
N_{\mathrm{i}}(\mathrm{HFS})=N_{\mathrm{i}}(30 \mathrm{~cm})-B G R t_{\mathrm{e}},
$$

with $B G R$ being the background rate and $t_{\mathrm{e}}$ being the emptying time of $35 \mathrm{~s}$. For the spin-flipped HFS, we obtain

$$
N_{\mathrm{sp}}(\mathrm{HFS})=\sum_{t_{\mathrm{s}}}^{t_{\mathrm{i}}} N(t)(30 \mathrm{~cm})-B G,
$$

where $B G=B G R\left(t_{\mathrm{i}}-t_{\mathrm{s}}\right)$.

For the determination of the background rate, we used three different methods: (i) we took data at absorber position $0 \mathrm{~cm}$, (ii) we took data with closed valves, and (iii) we analyzed the counts after emptying. With all three methods, we obtained results in agreement with each other. The resulting background uniformly distributed over time was $(45 \pm 5) \mathrm{mHz}$. In a second period of data collection, we improved the shielding around the detector and obtained $(14 \pm 4) \mathrm{mHz}$. For the data taken at PSI, we did not analyze the HFS and thus did not measure or analyze the $B G R$.

\section{The storage-time constant $\tau_{\text {st }}$}

For the determination of the storage-time constant $\tau_{\mathrm{st}}$ and even more for the $\beta$ and $\eta$ parameters, the knowledge of the correct cleaning time is very important. As an example, we present here the procedure for the evaluation of the correct

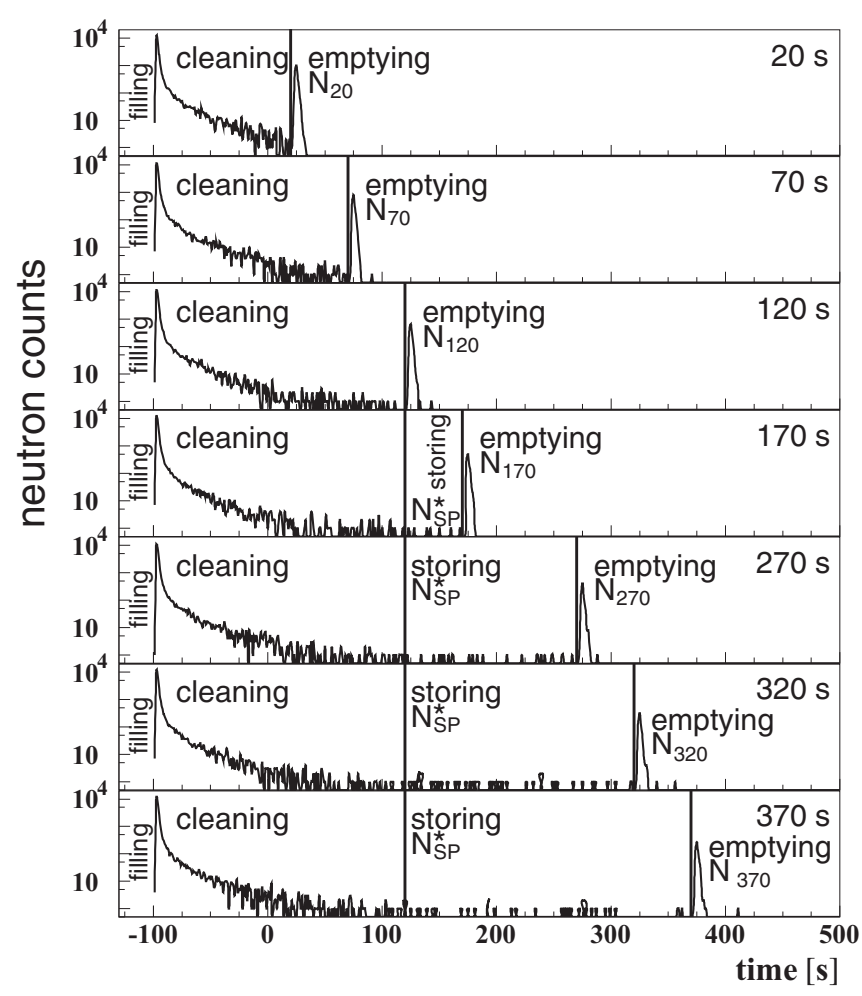

FIG. 12. Neutron counts with emptying from $t=20 \mathrm{~s}$ to $t=$ $370 \mathrm{~s}$. The sample is NiMo $88 / 12$, with absorber height $90 \mathrm{~cm} . N_{\mathrm{sp}}^{*}$, spin-flipped LFS plus half of the spin-flipped HFS plus background during storing, $N_{\mathrm{sp}}^{*}=N_{\mathrm{sp}}(\mathrm{LFS})+0.5 N_{\mathrm{sp}}(\mathrm{HFS})+B G ; N_{\mathrm{i}}, \mathrm{UCN}$ in the emptying peak after storage for $t_{\mathrm{i}}=20,70,120,170,270,320$, and $370 \mathrm{~s}$. For the determination of $\beta$ and $\eta$ values, the data at $t_{\mathrm{i}}=20$ and $70 \mathrm{~s}$ were not used.

cleaning time with the sample NiMo88/12 at an absorber height of $90 \mathrm{~cm}$. We applied this procedure for all samples and absorber heights.

In Fig. 12, data are shown for emptying counts at $t=20 \mathrm{~s}$ to $t=370 \mathrm{~s}$. After the respective background subtraction, the counts in the emptying peak, $N_{\mathrm{i}}$, are used to determine the storage-time constant, $\tau_{\mathrm{st}}$. This was obtained by fitting an exponential with two free parameters, $N_{0}$, the number of UCN at $t=0 \mathrm{~s}$, and $\tau_{\mathrm{st}}$, the storage-time constant, to the respective $N_{\mathrm{i}}$ values

$$
N_{\mathrm{i}}=N_{0} e^{-\frac{t}{\tau_{\mathrm{st}}}}
$$

In a first step, we used emptying times $t_{\mathrm{i}}$ at $t=20 \mathrm{~s}$ to $t=370 \mathrm{~s}$ in the fit. The values obtained were $\tau_{\mathrm{st}}=(266.1 \pm$ $4.0) \mathrm{s}$, and $N_{0}=2509.6 \pm 29$.9. The reduced $\chi^{2}$ of the fit was 2.9. We suspected that the bad $\chi^{2}$ might originate from the imperfect cleaning time of $100 \mathrm{~s}$ only. Therefore, in a second step, we omitted in the fit the data at $t_{20}$ and $t_{70}$ and obtained $\tau_{\mathrm{st}}=(282.2 \pm 7.6) \mathrm{s}, N_{0}=2364.5 \pm 59.4$ with a reduced $\chi^{2}$ of 0.32 .

In a last step, in order to estimate a time constant for the last part of the cleaning, $\tau_{\mathrm{cl}}$, we fixed these two values, fitted an additional exponential to the data at all $t_{\mathrm{i}}(20$ to $370 \mathrm{~s})$,

$$
N_{\mathrm{i}}=N_{\mathrm{cl}} e^{-\frac{t}{\tau_{\mathrm{cl}}}}+2364.5 e^{-\frac{t}{282.2}},
$$




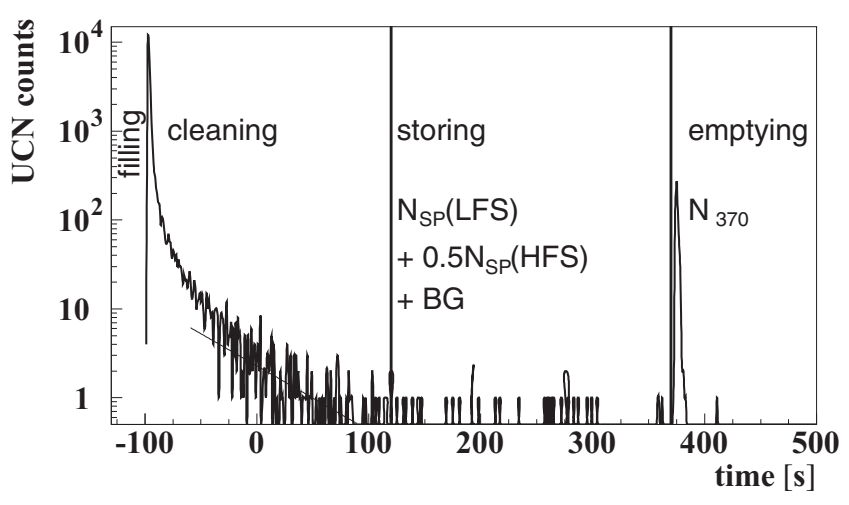

FIG. 13. Time spectrum of UCN for a holding time of 370$120=250 \mathrm{~s}$. The sample is NiMo88/12 and the absorber height is $90 \mathrm{~cm}$. An exponential was fitted to the data for $t=-20 \mathrm{~s}$ to $t=$ $+40 \mathrm{~s}$ with the result $\tau_{\mathrm{cl}}=(59 . \pm 13.8) \mathrm{s}$. This is appreciably smaller than the storage-time constant $\tau_{\mathrm{st}}=(282.2 \pm 7.6) \mathrm{s}$. Therefore, in the analysis, the spectral cleaning was increased from $100 \mathrm{~s}$ to 220 s, i.e., from $t=-100 \mathrm{~s}$ to $t=+120 \mathrm{~s}$. and obtained $\tau_{\mathrm{cl}}=36.8 \pm 12.5 \mathrm{~s}$ with $N_{\mathrm{cl}}=400 \pm 138$. The reduced $\chi^{2}$ here was 0.2 .

We have compared these values for $\tau_{\mathrm{cl}}$ to a direct analysis of the cleaning time constant in dedicated runs. One can see, e.g., from Fig. 13 that the cleaning does not follow one single exponential between $t=-100 \mathrm{~s}$ and $t=+100 \mathrm{~s}$. From $t=-20 \mathrm{~s}$ to $t=+40 \mathrm{~s}$ we have, however, fitted one single exponential,

$$
N_{\mathrm{j}}=N_{\mathrm{cl}} e^{-\frac{t}{\tau_{\mathrm{cl}}}} \text {. }
$$

As result, we obtain $\tau_{\mathrm{cl}}=59.9 \pm 13.8 \mathrm{~s}$. The $\chi^{2}$ of this fit was 59.3 for 58 degrees of freedom, leading to a reduced $\chi^{2}$ of 1.02. This $\tau_{\mathrm{cl}}$ value is in agreement with the result obtained just above. ${ }^{4}$

The storage-time constant for the NiMo88/12 sample at the $90-\mathrm{cm}$ absorber position is $\tau_{\mathrm{st}}=282.2 \mathrm{~s}$, i.e., a factor of 4.7 to 7.7 higher than $\tau_{\mathrm{cl}}$. This is a clear indication that the

\footnotetext{
${ }^{4}$ The cleaning has at least two different time constants: (i) wrong spin states (rather short $\tau_{\mathrm{cl}}$ ) and (ii) total energies $E>1.91 \mu_{\mathrm{N}} B=$ $1.91 \mu_{\mathrm{N}} 1.52 \mathrm{~T} \approx 90 \mathrm{neV}$, with $\tau_{\mathrm{cl}} \approx 60 \mathrm{~s}$.
}

TABLE III. Summary of results for storage-time constants $\tau_{\text {st }}$, depolarization probability per wall collision $\beta$, and loss parameter $\eta$; $z_{\mathrm{a}}$ is the absorber position. We have averaged the HFS data at 20 and $30 \mathrm{~cm}$ for the sake of improved statistics.

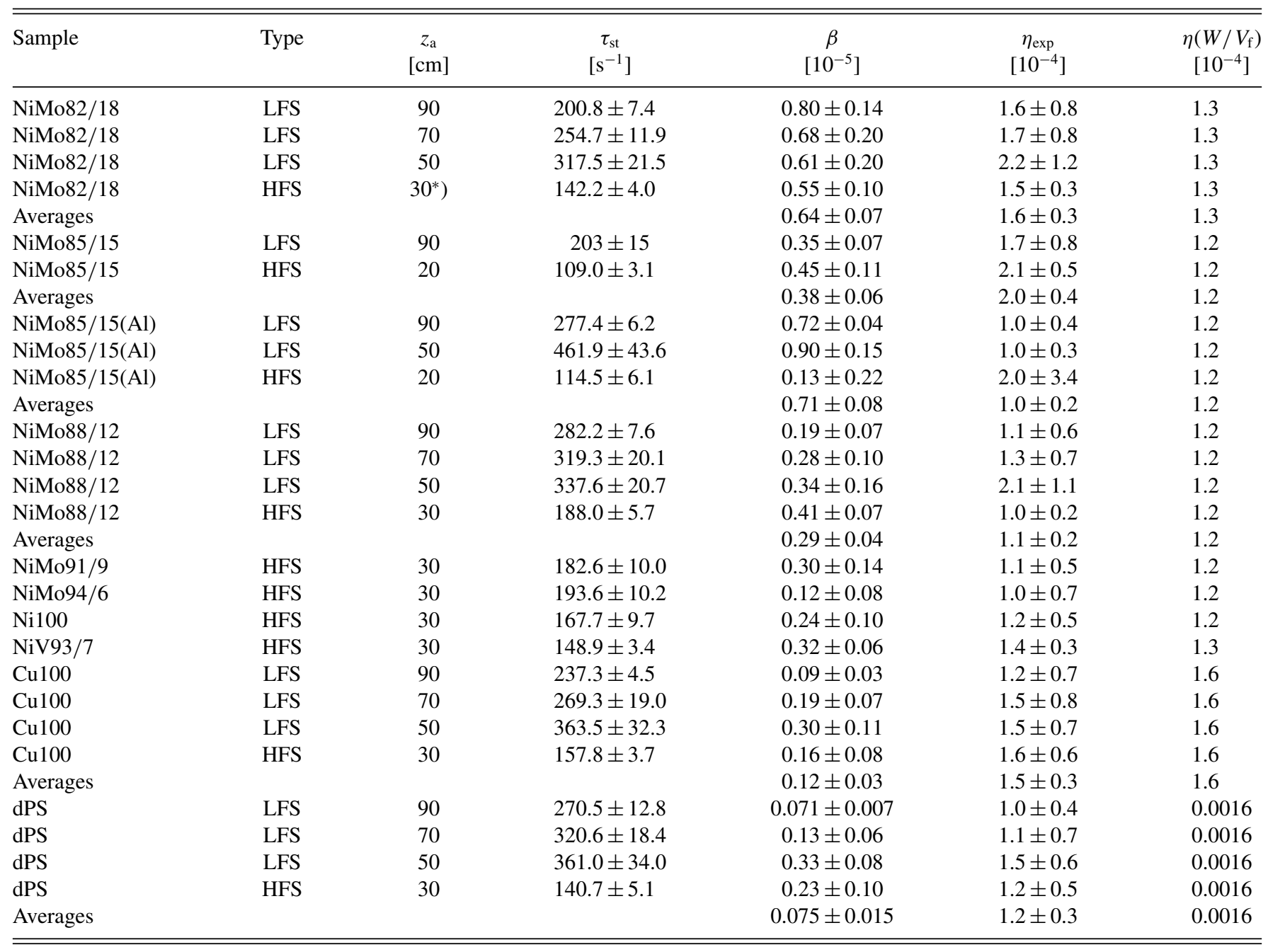




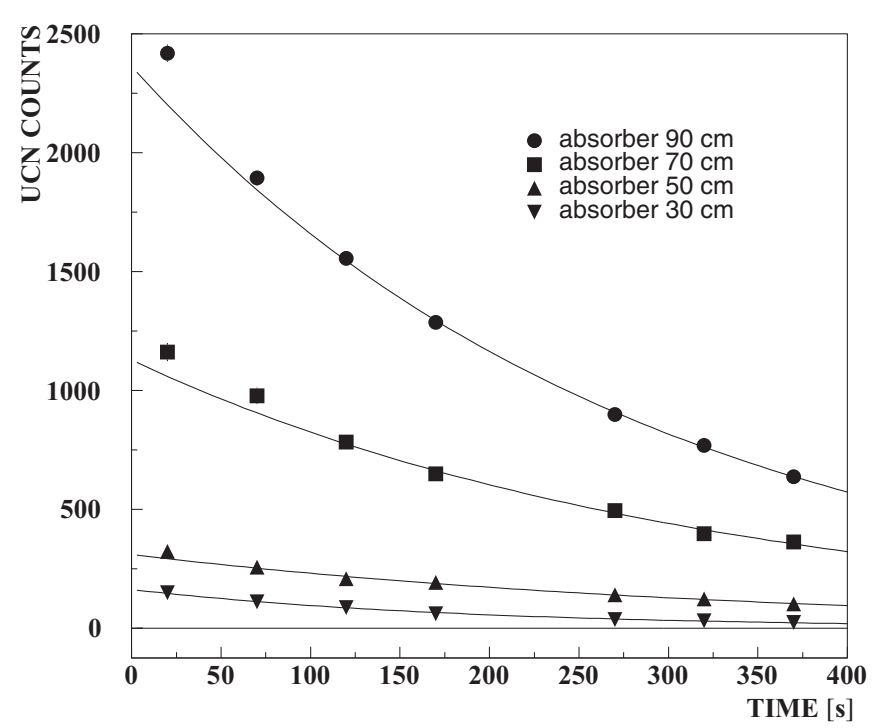

FIG. 14. Determination of the storage-time constants for NiMo88/12 and different absorber positions. The storage-time constants are obtained from the $N_{\mathrm{i}}$ values after different holding times ranging from $t_{\mathrm{i}}=20$ to $t_{\mathrm{i}}=370 \mathrm{~s}$. The background originating from HFS and ambient events was subtracted. For absorber positions 90 and $70 \mathrm{~cm}$, the data at 20 and $70 \mathrm{~s}$ were not included in the fit. For absorber positions $50 \mathrm{~cm}$, the data at $20 \mathrm{~s}$ was not included in the fit. At absorber position $30 \mathrm{~cm}$, there are only HFS events and background; cf. Figs. 6 and 12. Here, the background measured with absorber position $z_{\mathrm{a}}=0 \mathrm{~cm}$ was subtracted.

majority of the $N_{\mathrm{sp}}$ events for $t_{\mathrm{i}}<120 \mathrm{~s}$ do not originate from spin flips but are cleaning events. Further indication for the duration of the spectral cleaning longer than $100 \mathrm{~s}$ is presented in Sec. VI A below. As a result of this study, the cleaning times in the analysis were fixed to (i) $220 \mathrm{~s}$ for absorber positions 70 to $90 \mathrm{~cm}$, as indicated in Fig. 12, (ii) to $170 \mathrm{~s}$ for absorber positions 40 to $60 \mathrm{~cm}$, and (iii) to $100 \mathrm{~s}$ for absorber positions of $z_{\mathrm{a}} \leqslant 30 \mathrm{~cm}$.

The $\tau_{\mathrm{st}}$ results with correct cleaning times for all absorber positions of the NiMo88/12 sample are presented in Fig. 14 and Table III. The reduced $\chi^{2}$ values of the fits were 0.32 , $0.71,0.46$, and 1.1 for the four absorber positions, $90,70,50$, and $30 \mathrm{~cm}$. In Fig. 14, one can see that for absorber positions 90 and $70 \mathrm{~cm}$ and $t=20 \mathrm{~s}$ and $t=70 \mathrm{~s}$ the data are clearly above the exponential line and therefore were not used for the determination of $\tau_{\mathrm{st}}$. The $\tau_{\mathrm{st}}$ values for all samples and absorber positions are shown in Table III.

The storage-time constants for the sample NiMo82/18 as a function of absorber position are shown in Fig. 15. There is a clear tendency for smaller absorber heights, i.e., for smaller frequencies of wall collisions, that the $\tau_{\mathrm{st}}$ values are higher, as expected. This holds for all samples. For the HFS, the collision frequencies are higher: Because of the high gradient of the magnetic field, $d \vec{B}_{\perp} / d r \approx 16.6 \mathrm{~T} / \mathrm{m}$, in the region of the air gap of the H-type split-coil magnet, HFS with low energies are reflected by the optical wall potential only to a very short distance before they are pulled back again toward the sample wall by the strong gradient of the magnetic field. High-fieldseeking UCN with kinetic energies higher than about $33 \mathrm{neV}$

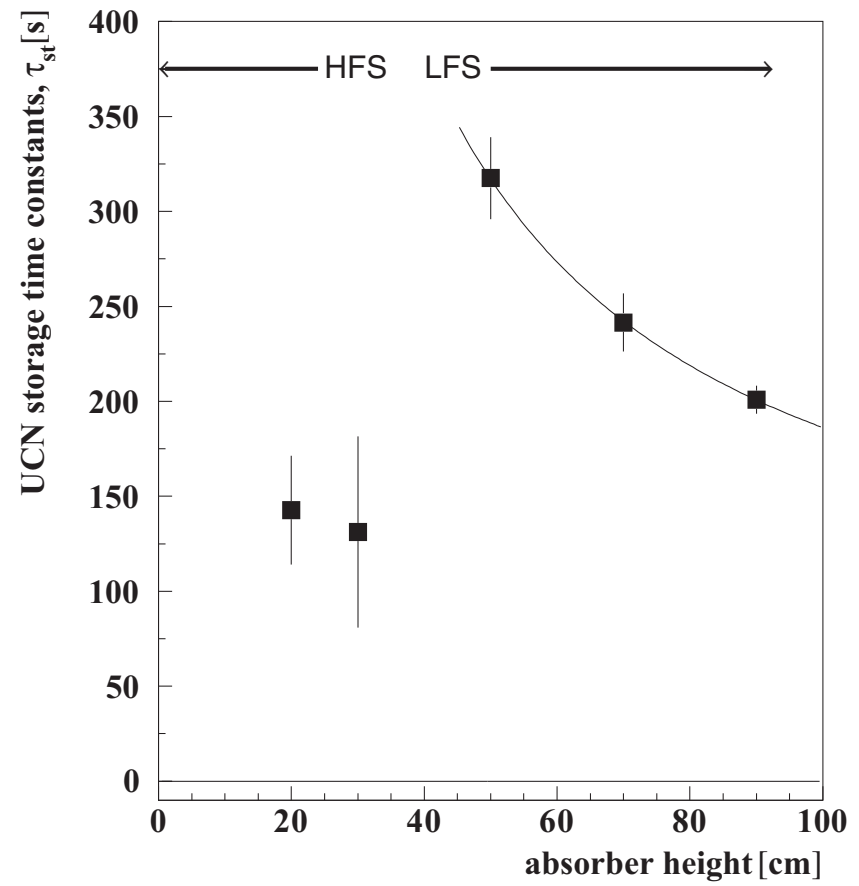

FIG. 15. Storage-time constants for NiMo82/18 at different absorber positions. The solid line is a fit to the data, $\tau_{\mathrm{st}}=A / z+B$, with $A=13094 \pm 2563$ and $B=55 \pm 30$, with $z$ being the variable for the absorber height.

are reflected toward $r=0 \mathrm{~cm}$ and then accelerated toward the opposite side of the sample.

\section{RESULTS}

\section{A. The depolarization probability $\beta$}

The depolarization probability per wall collision $\beta$ for one energy is derived from Eq. (10):

$$
\beta=\frac{N_{\mathrm{sp}}}{\left(N_{\mathrm{s}}-N_{\mathrm{i}}\right) \tau_{\mathrm{st}} \nu} .
$$

Here, $N_{\mathrm{sp}}$ is the number of spin-flipped UCN during storage, $N_{\mathrm{s}}$ is the number of UCN at the beginning of the UCN storage, and $N_{\mathrm{i}}$ is the number of $\mathrm{UCN}$ at the end of the storage, respectively.

The depolarization probability per wall collision is assumed to be independent of energy. This assumption is supported by (i) the results of Refs. $[48,49]$ and (ii) from the fact that $\tau_{\mathrm{st}}$ is inversely proportional with absorber height, i.e., energy, cf. Fig. 15, while the frequency of wall collisions for absorber height $z>30 \mathrm{~cm}$ is approximately linear with $z$; see Fig. 9 .

Equation (30) is strictly valid for monoenergetic UCN only. In order to take into account the UCN spectrum stored in the sample, Eq. (30) is modified to

$$
\beta=\frac{N_{\mathrm{sp}}}{\left(N_{\mathrm{s}}-N_{\mathrm{i}}\right) \tau_{\mathrm{st}}\langle v\rangle},
$$

with $\langle v\rangle$ from Eq. (11).

For the correct determination of the $\beta$ parameter, the precise knowledge of backgrounds is crucial. Besides the backgrounds 


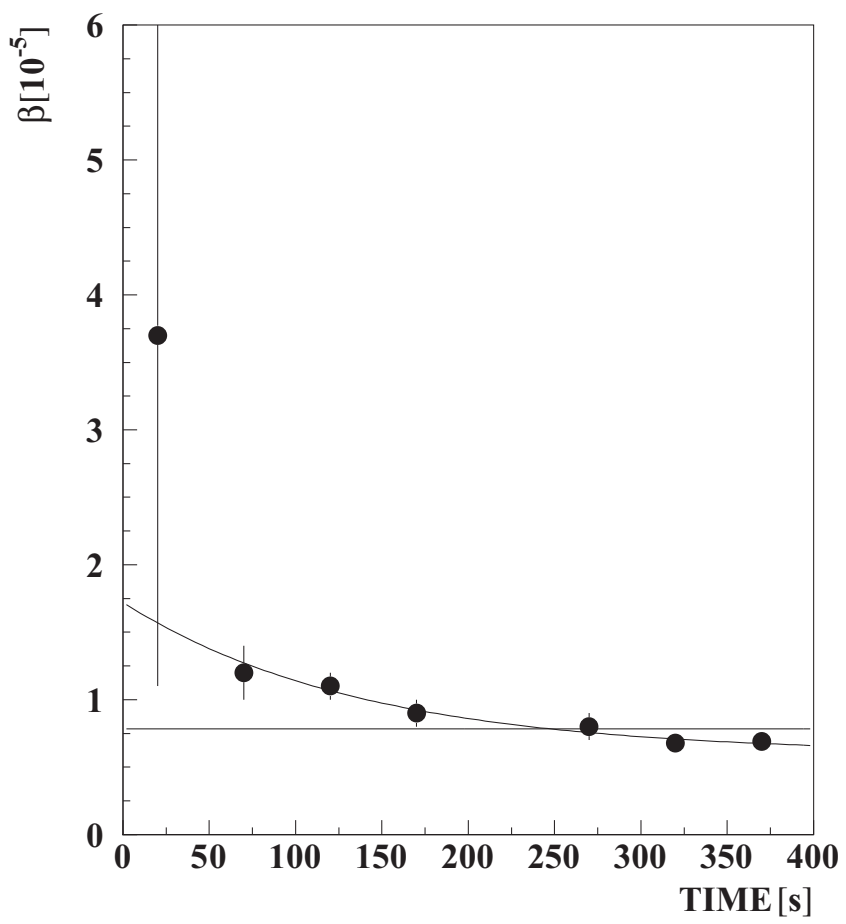

FIG. 16. Calculated values of $\beta_{100}$ from measured data vs $t$ for a spectral cleaning time of $100 \mathrm{~s}$. The sample is NiMo88/12 and the absorber position is $90 \mathrm{~cm}$. One can clearly see a dependance in time. This is supported by fitting a constant to the data points (see horizontal line). One obtains $\beta_{100}=(0.78 \pm 0.03) \times 10^{-5}$ with a reduced $\chi^{2}$ of 3.7. Fitting an exponential, $y=a+b \exp \frac{t}{\tau_{\mathrm{cl}}}$ in order to take into account imperfect cleaning, one obtains $\tau_{\mathrm{cl}}=(137 \pm 107)$ $\mathrm{s}$, in agreement with the $\tau_{\mathrm{cl}}$ values obtained in Sec. V D. The reduced $\chi^{2}$ of this fit is 0.25 .

described in Sec. V C, the spectral cleaning time of the stored $\mathrm{UCN}$ is of utmost importance as we demonstrate in the following.

Figure 16 shows the calculated $\beta$ parameter from the measured data of the NiMo88/12 sample and an absorber height of $90 \mathrm{~cm}$ with a cleaning time of only $100 \mathrm{~s}$, as was suggested earlier $[49,82]$. The mean value here is $\beta_{100}=$ $(0.78 \pm 0.03) \times 10^{-5}$. The reduced $\chi^{2}$ of the fit is as large as 3.7. Since we always have a spectrum of different kinetic energies stored in the sample, one might argue that the relatively faster $\mathrm{UCN}$ depolarize faster. Then, the $\beta$ values at lower absorber positions should show an energy-dependent effect. This behavior was, however, not found; see below. In order to account for incomplete spectral cleaning, we thus have fitted an exponential to the data in Fig. 16 and find $\tau_{\mathrm{cl}}=(137 \pm 107) \mathrm{s}$, in agreement with the $\tau_{\mathrm{cl}}$ values found in Sec. V D above.

In Fig. 17, we present the $\beta$ values with the correct cleaning time of $220 \mathrm{~s}$ as a function of the holding times $t_{\mathrm{i}}$. Here, the mean value is $\beta_{220}=(0.19 \pm 0.07) \times 10^{-5}$. The reduced $\chi^{2}$ of this fit is 0.34 . The small value of $\chi^{2}$ is due to correlation between the single values: the storage-time constant $\tau_{\mathrm{st}}, N_{\mathrm{s}}$, and $\langle v\rangle$ are the same at all $t_{\mathrm{i}}$.

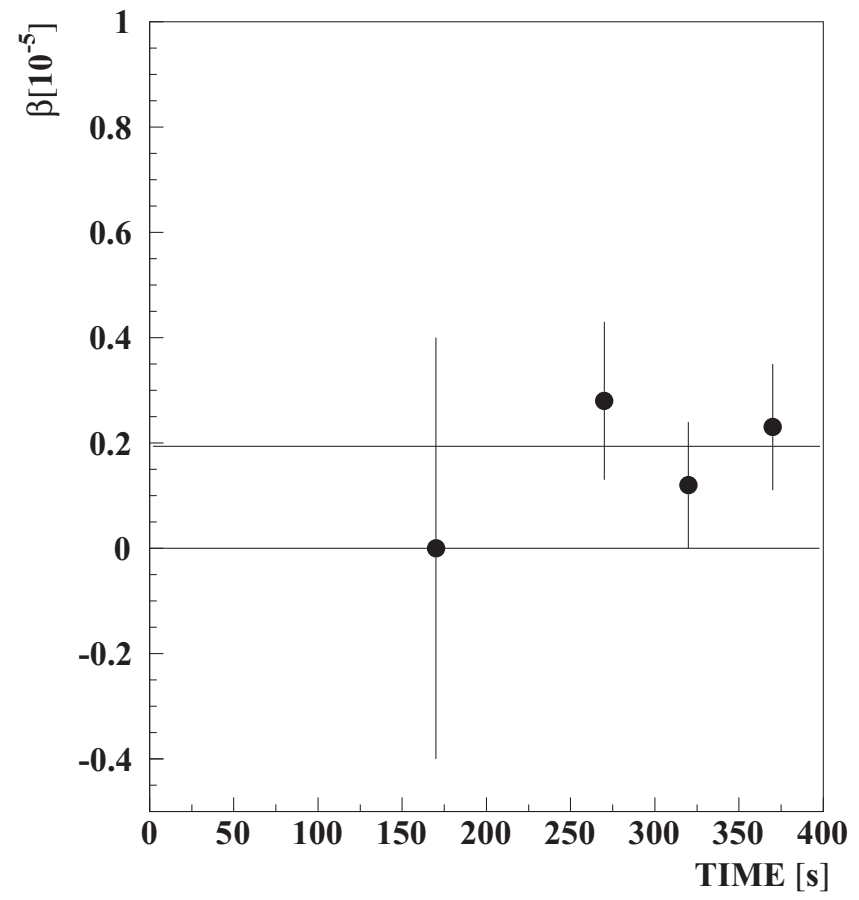

FIG. 17. The depolarization parameter $\beta_{220}$ for a spectral cleaning time of $220 \mathrm{~s}$. The sample is NiMo88/12 and the absorber position is $90 \mathrm{~cm}$. Here, the mean value is $\beta_{220}=(0.19 \pm 0.07) \times 10^{-5}$.

The $\beta$ values as a function of absorber position for the paramagnetic samples NiMo82/18, NiMo85/15, and NiMo88/12 on glass substrates are shown in Fig. 18. There is no dependance on absorber height or kinetic energy as could have been suggested from Fig. 16.

In Fig. 19, we show the $\beta$ values averaged over all holding times for an absorber height of $90 \mathrm{~cm}$ and the average over all absorber heights as a function of the molybdenum content in NiMo alloys. Here, the depolarization decreases with decreasing molybdenum content. This is somewhat in contrast to expectations deduced from Refs. [57] and [89], where it was claimed that "the total magnetic moment of the alloy also decreases as Mo concentration increases and finally vanishes at $x \simeq 0.15$ " (p. 644). Here, $x$ is the molybdenum content in a NiMo alloy. At lower absorber positions, this behavior is similar but less visible due to the lower statistical accuracy. The results for all samples and absorber heights are given in Table III.

\section{HFS and ferromagnetic samples}

According to measurements of the Curie temperature of NiMo alloys (see, e.g., Ref. [43]), one would expect ferromagnetic behavior of these alloys at $300 \mathrm{~K}$, i.e., room temperature for Mo contents of less than 5.4 at.\%. Another reference [57] suggests ferromagnetic behavior of NiMo alloys at room temperature for a Mo content of less than 9.6 at.\%. In a third paper [89], the Curie temperature is $300 \mathrm{~K}$, for a Mo content of 7 at. $\%$.

Since the material optical potential, $V_{\mathrm{f}}$, increases with decreasing Mo content (see Table I), the number of transportable 


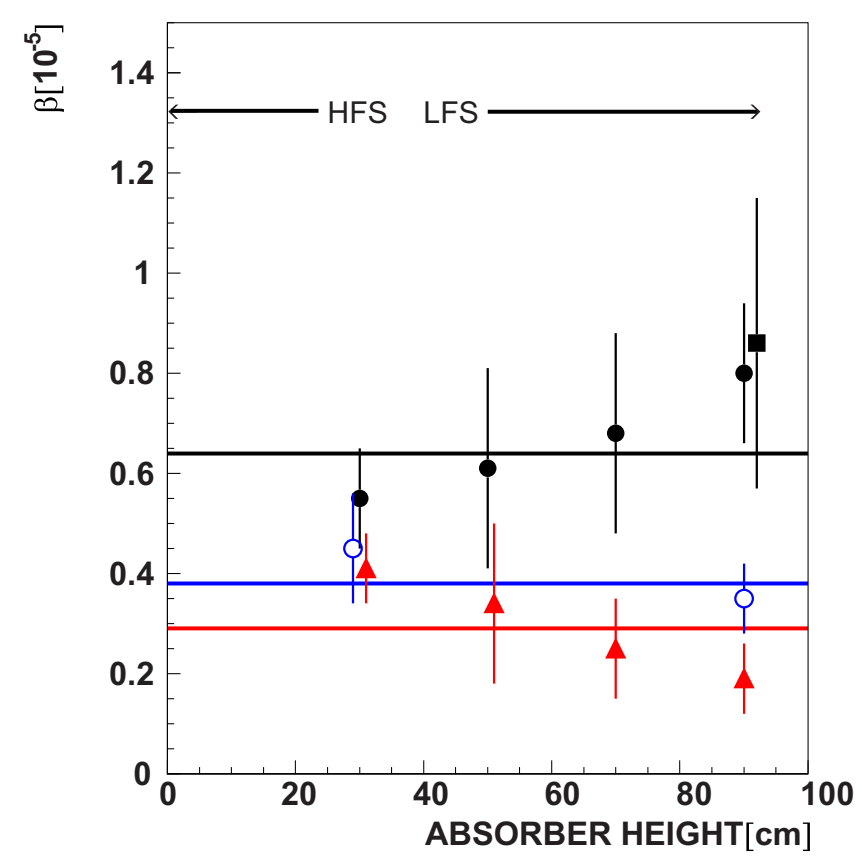

FIG. 18. Depolarization probability $\beta$ for the paramagnetic NiMo alloys and for different absorber positions. (i) NiMo82/18, black circles, data taken at the ILL; black square, data taken at PSI. The average value (black line, upper line) is $\beta=(0.64 \pm 0.07) \times 10^{-5}$ with a reduced $\chi^{2}$ of 0.73 . (ii) NiMo85/15, blue open circles. The average value (blue line, middle line) is $\beta=(0.38 \pm 0.06) \times 10^{-5}$ with a reduced $\chi^{2}$ of 0.59 . (iii) NiMo88/12, red triangles. The average value (red line, lower line) is $\beta=(0.29 \pm 0.04) \times 10^{-5}$ with a reduced $\chi^{2}$ of 1.74. HFS, high-field seekers; LFS, low-field seekers.

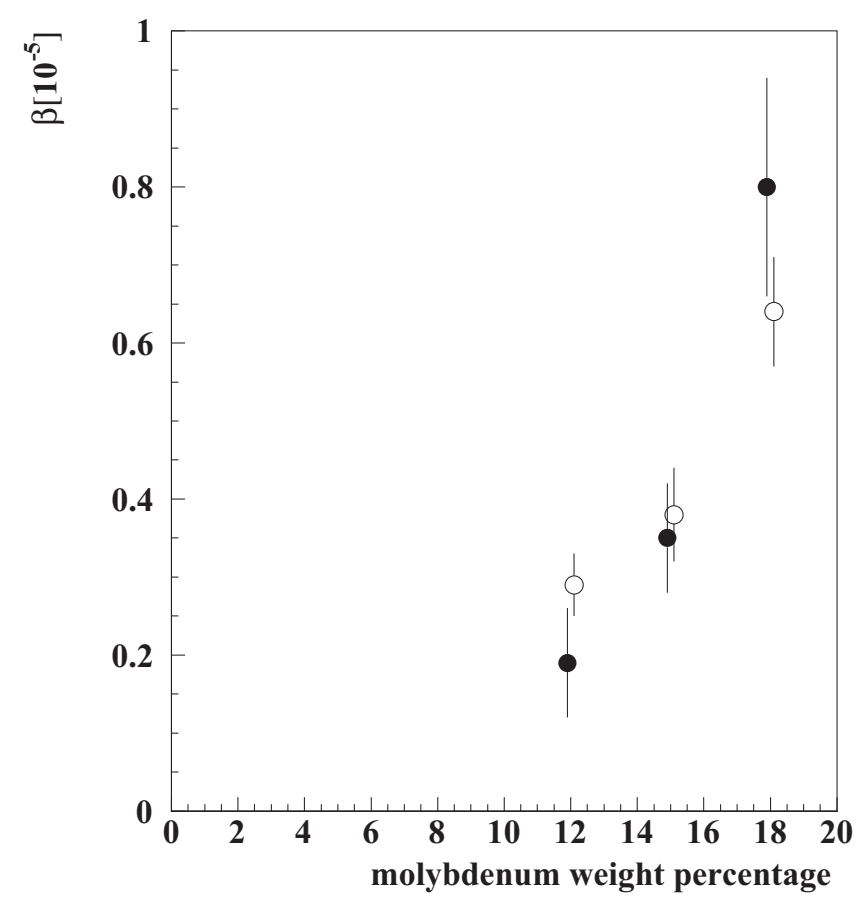

FIG. 19. The depolarization parameter $\beta$ as a function of the molybdenum content in NiMo alloys, (i) averaged over all holding times, $t_{\mathrm{i}}$, for absorber heights $90 \mathrm{~cm}$ (full dots) and (ii) averaged over all holding times and absorber heights (open circles).

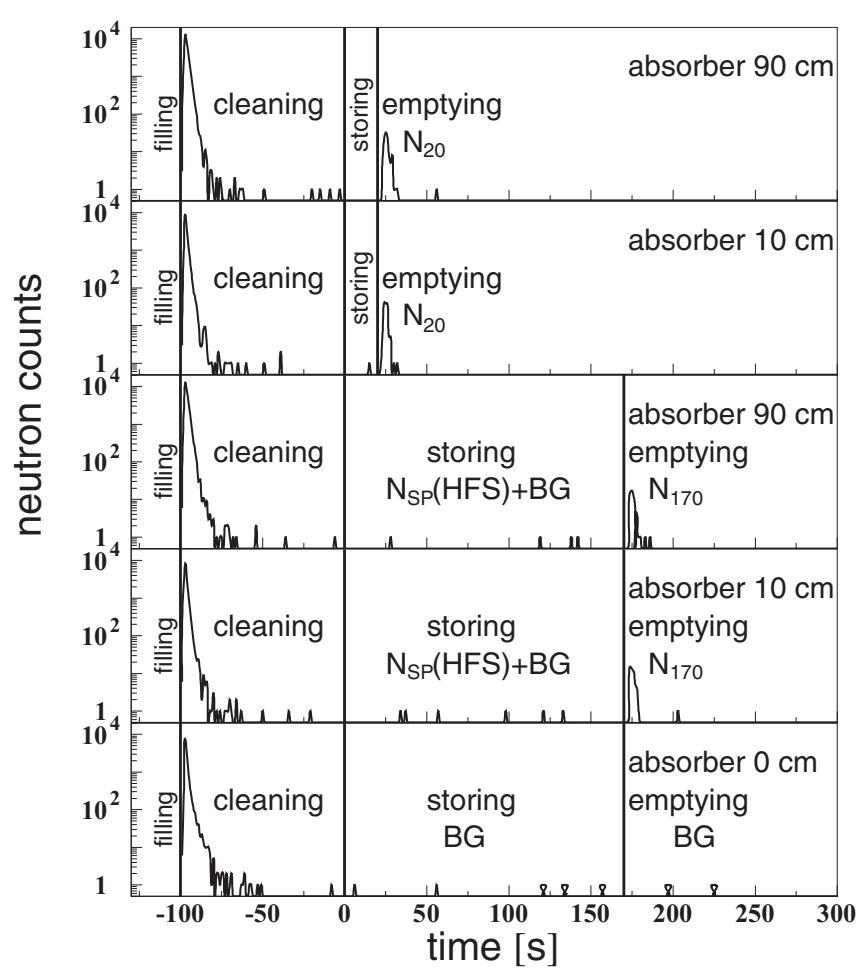

FIG. 20. Detector counts during data taking at absorber positions of 90,10 , and $0 \mathrm{~cm}$. The sample is Ni100.

UCN in such guides also increases. In order to clarify the ambiguity of the results in Refs. $[43,57,89]$ and to find out the optimal conditions for the transport of polarized UCN, we also investigated NiMo alloys with weight percentages of 91/9, 94/6, and 100/0.

Figure 20 shows the UCN counts over time for Ni100 and absorber positions at 90,10 , and $0 \mathrm{~cm}$. First, one can see that the cleaning time of $100 \mathrm{~s}$, i.e., from -100 to $0 \mathrm{~s}$, is enough here for all absorber positions. Second, the counts in the emptying peaks for absorber positions 90 and $10 \mathrm{~cm}$ agree fairly well with each other. The numbers are $N_{20}=$ $(116.9 \pm 7.8)$ for $90 \mathrm{~cm}$ and $N_{20}=(119.5 \pm 3.9)$ for $10 \mathrm{~cm}$. At 170-s holding time, the numbers are $N_{170}=(57.9 \pm 5.4)$ and $N_{170}=(49.9 \pm 2.5)$ for 90 and $10 \mathrm{~cm}$, respectively. The slight difference at $t=170 \mathrm{~s}$ may originate from the fact that at $10 \mathrm{~cm}$ some of the stored HFS are absorbed: Unfortunately, this absorber position in the experiment was slightly too low and thus the absorber cuts into the energy distribution of the HFS. Finally, the events in the cleaning peak are about 2000 counts more than in the cleaning peaks of the paramagnetic samples. This difference corresponds fairly well to the emptying counts at $t=20 \mathrm{~s}$ of the paramagnetic samples. All these observations indicate that in the ferromagnetic samples no LFS were stored: They depolarize instantaneously and are registered in the detector already during cleaning. The equal counts at absorber positions 90 and $10 \mathrm{~cm}$ prove there are only HFS stored in this sample. The samples NiMo94/6, 91/9, and NiV93/7 show the same behavior. Thus, in our experiment, $9 \mathrm{wt} . \%$ of $\mathrm{Mo}$ in $\mathrm{Ni}$ corresponding to 6.5 at.\% is still ferromagnetic, while $12 \mathrm{wt} . \%$ corresponding to 8.7 at.\% is paramagnetic. Our results are in 
good agreement with that of Ref. [89] while the agreement with Refs. [43,57] is poor. The results of the different parameters for the ferromagnetic samples are also shown in Table III.

The phenomenon of depolarization of the LFS in ferromagnetic materials at magnetic holding fields below saturation and the storage of HFS in strong magnetic fields above saturation was described in Ref. [76]. Here, we give a short general outline in order to make the paper self-contained.

For the stored HFS, the depolarization rate is very small and almost equal to the background. At the inner radius of the cylindrical vertical neutron guide, i.e., where the material under investigation was coated onto the cylinder wall, the magnetic field in the midplane of the air gap, $z=0, r=35 \mathrm{~mm}$, is about $2.1 \mathrm{~T}$. At this field value, the ferromagnetic Ni coating is completely in saturation with the relative permeability $\mu=1$ for $B>0.6 \mathrm{~T}$. The magnetic field gradient here is $d \vec{B}_{\perp} / d x \approx 16.6 \mathrm{~T} / \mathrm{m}$.

The spin of UCN, traveling in a nonhomogeneous field, follows the change of the magnetic field, as long as the adiabatic condition is fulfilled. That is, the motion of the neutron is so slow that its magnetic moment always keeps the same orientation with respect to the magnetic field. The condition for this is that the time variation, i.e., the rotation of the magnetic field $B$ seen by the neutron as it moves through the field is much less than the Larmor frequency, $\omega_{\mathrm{L}}$, of the neutron spin:

$$
\frac{\left|d \vec{B}_{\perp} / d t\right|}{B}=\frac{\left|d \vec{B}_{\perp} / d x\right||d x / d t|}{B} \ll \omega_{\mathrm{L}}=\frac{2 \mu_{\mathrm{n}}}{\hbar} B,
$$

with $d \vec{B}_{\perp} / d x$ being the magnetic field gradient perpendicular to $\vec{B},|d x / d t|$ being the neutron velocity, and $\mu_{\mathrm{n}}$ being the neutron magnetic moment, $\mu_{\mathrm{n}}=-1.91 \mu_{\mathrm{N}}$.

At $E_{\text {kin }}(\max )=100 \mathrm{neV}$, the velocity of UCN is $v_{\mathrm{n}}(\max )$ $\approx 4.4 \mathrm{~m} / \mathrm{s}$. For the HFS, however, the average velocity is more like $v_{\mathrm{n}}(\mathrm{av})=3 \mathrm{~m} / \mathrm{s}$ and $\left(d \overrightarrow{B_{\perp}} / d t\right) / B=15.8 \mathrm{~Hz}$. The Larmor frequency is $\omega_{\mathrm{L}} \approx 4 \times 10^{8} \mathrm{~Hz}$. That is, the adiabatic condition, Eq. (32), for the orientation of the neutron spin to follow the magnetic field lines, is well fulfilled for the HFS. The strong magnetic field, both in the scatter material and inside the sample tube, is parallel to the $z$ axis, see Fig. 2, as is the spin orientation of the neutrons. From a measurement at different absorber heights, one can see that all stored HFS in a magnetic sample oscillate and are confined within $z \approx 10 \mathrm{~cm}$ above and $z \approx 20 \mathrm{~cm}$ below the vertical center of the dipole field in the air gap of the magnet; see Fig. 20. That is, at absorber position $z=0 \mathrm{~cm}$, no stored $\mathrm{UCN}$ were counted.

In the case of changing the magnetic field from 1.45 to $1.52 \mathrm{~T}$ in a split second $(\sim 0.3 \mathrm{~s})$ during (or after) the cleaning period the ratio of $\left(d \overrightarrow{B_{\perp}} / d t\right) / B$ will not change. Thus, the adiabatic condition, cf. Eq. (32), is well fulfilled and no depolarization can occur due to this change.

Above $z \approx 130 \mathrm{~mm}$, the magnetic field in the magnet bore drops below saturation in the ferromagnetic Ni coatings $(B<0.6 \mathrm{~T})$ and therefore the relative permeability of the ferromagnetic coating increases to $\mu>1$. As a consequence, the stray magnetic field entering the ferromagnetic coating is suddenly strongly bent and has a discontinuous distribution and gradient. The adiabatic condition is then no longer fulfilled and all neutrons above this point, i.e., the LFS, are instantaneously depolarized and fall through the magnetic field into the detector.

\section{B. The wall loss parameter $\eta$}

As mentioned above, the wall loss coefficient $\eta$ is the ratio of the imaginary part $W$ and the real part $V_{\mathrm{f}}$ of the material optical potential; see Eq. (2). Experimentally, we can deduce $\eta$ from the average losses over angle $\bar{\mu}(E)$ given by Eq. (6). This equation holds for monoenergetic UCN with energy $E$. In our apparatus, we have, however, a UCN kinetic energy distribution between 0 and $\sim 60 \mathrm{neV}$ and thus we obtain from Eq. (7)

$$
\left\langle\frac{1}{\tau_{\mu}}\right\rangle\left(E_{\max }\right)=\langle\mu \nu\rangle=\frac{1}{\tau_{\mathrm{st}}}-\frac{1}{\tau_{\mathrm{n}}}-\left\langle\frac{1}{\tau_{\beta}}\right\rangle .
$$

For the lifetime of the neutron, we use $\tau_{\mathrm{n}}=(880.3 \pm$ 1.1)s [90]. Since our measured storage-time constants are significantly shorter than the neutron lifetime, the $\mu$ and $\eta$ values are not altered significantly by a change of the neutron lifetime of several seconds [91-95].

From Eqs. (33) and (6), one obtains

$$
\langle\mu \nu\rangle=\frac{\int_{0}^{H} \int_{E_{\min }}^{E_{\max }} \bar{\mu}\left[E_{0}-V(z)\right] \frac{d v}{d z}\left(E_{0}, z\right) g\left(E_{0}\right) d E_{0} d z}{\int_{E_{\min }}^{E_{\max }} g\left(E_{0}\right) d E_{0}}
$$

with $g_{\mathrm{i}}\left(E_{0}\right)$ from Fig. 7 , line through open squares.

Using numerical integration, we find

$$
\eta_{\exp }=\frac{\left[\frac{1}{\tau_{\mathrm{st}}}-\frac{1}{\tau_{\mathrm{n}}}-\langle\beta \nu\rangle\right] \sum_{\mathrm{i}}^{\mathrm{i}\left(E_{0}\right)} g_{\mathrm{i}}\left(E_{0}\right)}{2 \sum_{\mathrm{i}=1}^{\mathrm{i}\left(E_{0}\right)}\left\{\left[\frac{V_{\mathrm{f}}}{E_{\mathrm{i}}} \arcsin \left(\frac{E_{\mathrm{i}}}{V_{\mathrm{f}}}\right)^{1 / 2}-\left(\frac{V_{\mathrm{f}}}{E_{\mathrm{i}}}-1\right)^{1 / 2}\right] \nu_{\mathrm{i}} g_{\mathrm{i}}\left(E_{0}\right)\right\}} .
$$

Here, $E_{\mathrm{i}}$ is the maximum kinetic energy, a LFS can have for a given absorber position $z_{\mathrm{i}}$, e.g., $\sim 60 \mathrm{neV}$ for $90 \mathrm{~cm}, \sim 40 \mathrm{neV}$ for $70 \mathrm{~cm}, \sim 20 \mathrm{neV}$ for $50 \mathrm{~cm}$, and $\sim 0 \mathrm{neV}$ for $30 \mathrm{~cm}$.

For the HFS, the maximum kinetic energy does not depend on absorber position. As in Sec. V B 2, we have performed a Monte Carlo simulation to evaluate their energy distribution. In detail, the simulation was done to evaluate the parameter $v^{*}$, where

$$
\frac{1}{v^{*}}=\frac{\sum_{\mathrm{i}} g_{\mathrm{i}}^{\prime}\left(E_{\mathrm{i}}\right)}{2 \sum_{\mathrm{i}}\left\{\left[\frac{V_{\mathrm{f}}}{E_{\mathrm{i}}} \arcsin \left(\frac{E_{\mathrm{i}}}{V_{\mathrm{f}}}\right)^{1 / 2}-\left(\frac{V_{\mathrm{f}}}{E_{\mathrm{i}}}-1\right)^{1 / 2}\right] v_{\mathrm{i}} g_{\mathrm{i}}^{\prime}\left(E_{\mathrm{i}}\right)\right\}} ;
$$

cf. Eq. (35). However, now $g^{\prime}\left(E_{\mathrm{i}}\right)$ is the simulated distribution of the kinetic energies of the HFS at the instant of the collision with the sample wall. Here, we have assumed the part for diffused scattering to be 0.01 . Varying this parameter between 0.0 and 0.03 does not change the result significantly. The outcome of this Monte Carlo simulation is shown in Table IV. High-field-seeking UCN while bouncing at the sample walls have kinetic energies ranging from 0 to $100 \mathrm{neV}$. The shape of this $g^{\prime}$ distribution from the Monte Carlo simulation for the HFS is close to trapezoidal with side slopes from 0 to $10 \mathrm{neV}$ and 90 to $100 \mathrm{neV}$.

The $\eta$ values for both LFS and HFS for NiMo82/18 are displayed in Fig. 21. The results for all samples and 
TABLE IV. Results of the Monte Carlo simulation for the evaluation of the parameter $v^{*}$ of the HFS; cf. Eq. (36).

\begin{tabular}{lccc}
\hline \hline Material & Weight percentages & $\begin{array}{c}V_{\mathrm{f}} \\
{[\mathrm{neV}]}\end{array}$ & $\begin{array}{c}v^{*} \\
{\left[\mathrm{~s}^{-1}\right]}\end{array}$ \\
\hline NiMo & $82 / 18$ & 221.5 & $38.2 \pm 1.0$ \\
NiMo & $85 / 15$ & 225.4 & $37.8 \pm 1.0$ \\
NiMo & $88 / 12$ & 229.5 & $38.0 \pm 1.0$ \\
NiMo & $91 / 9$ & 223.5 & $39.1 \pm 0.9$ \\
NiMo & $94 / 6$ & 218.7 & $38.7 \pm 1.0$ \\
Ni & $100 / 0$ & 209.5 & $39.8 \pm 1.0$ \\
NiV & $93 / 7$ & 210.1 & $39.7 \pm 1.0$ \\
$\mathrm{Cu}$ & $100 / 0$ & 167 & $45.9 \pm 0.8$ \\
$\mathrm{dPS}$ & $100 / 0$ & 161 & $47.2 \pm 0.8$ \\
\hline \hline
\end{tabular}

absorber positions are listed in Table III. The experimental data are on average slightly higher than expected from $W / V_{\mathrm{f}}$; however, they are mostly in agreement within the uncertainties. In the case of dPS, where one expects an extremely low loss factor $\left(W / V_{\mathrm{f}}=0.0016 \times 10^{-4}\right)$, the difference is three orders of magnitude. This difference, previously termed anomalous losses, is attributed to, e.g., impurities, low-energy heating, surface contaminations or microscopic holes in the coating, and contaminations of the surface with hydrogen $[65-67,96,97]$. The data also show that there is no dependence on energy, as expected.

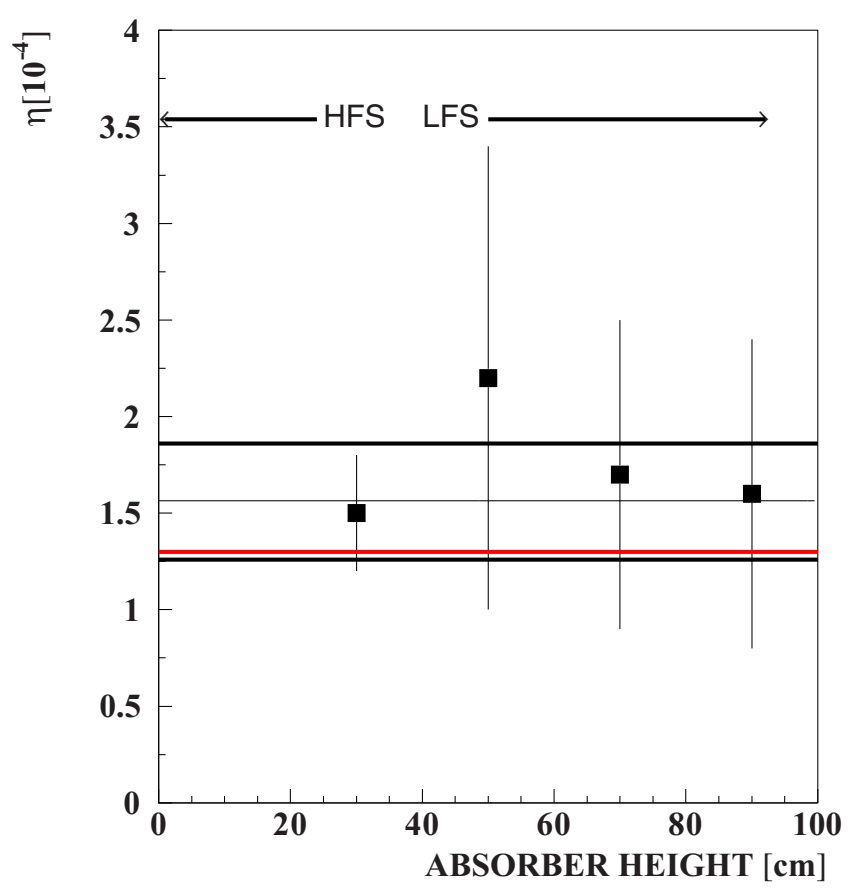

FIG. 21. Loss parameter of UCN $\eta$ over absorber height. The sample is NiMo82/18. A constant was fitted to the data, $(\eta=1.6 \pm$ $0.3) \times 10^{-4}$. The reduced $\chi^{2}$ of the fit is 0.2 . The small value of $\chi^{2}$ is partly due to correlation: The neutron lifetime and the optical potential are common to all single $\eta$ values at the different absorber heights. The red line (second horizontal line from below) is the theoretically expected value from $W / V_{\mathrm{f}}$.

\section{CONCLUSIONS}

Nickel molybdenum alloys are often used for the transport of polarized as well as unpolarized UCN. We have investigated the wall losses and depolarization probability per wall collision of the alloys NiMo82/18, NiMo85/15, NiMo88/12, NiMo91/9, NiMo94/6, Ni100, and NiV93/7. With a nickel content of $91 \mathrm{wt} \% \%$ or more, depolarization of the LFS happened spontaneously and only the HFS were stored in the region of the high magnetic field $(1.52 \mathrm{~T})$ in the vicinity of the air gap of the H-type split-coil magnet.

For all samples, we do not see an energy dependence for the $\beta$ and $\eta$ parameters; see, e.g., Figs. 18 and 21. The $\eta$ parameters over molybdenum content of the samples is shown in Fig. 22. No variation over the molybdenum content is visible.

All our experimental $\eta$ values agree with the theoretically expected values, $W / V_{\mathrm{f}}$, except for dPS where we establish anomalous losses at room temperature at a level of four standard deviations. It is, however, remarkable that $\eta(\mathrm{dPS})$ is more or less the same as that of the other materials investigated though its $W / V_{\mathrm{f}}$ is three orders of magnitude less.

The $\beta$ values of the paramagnetic NiMo alloys on glass substrates over molybdenum content, see Fig. 19, show an unexpected result: $\beta$ increases with increasing Mo content. This is in contradiction to expectations from the literature $[43,57,89]$, where a decreasing magnetic moment with increasing Mo content is reported. It should lead to a lower depolarization probability for higher Mo content. In order to confirm this effect, further measurements with different samples of the same alloys will be necessary. Furthermore, we find ferromagnetic behavior from our depolarization data

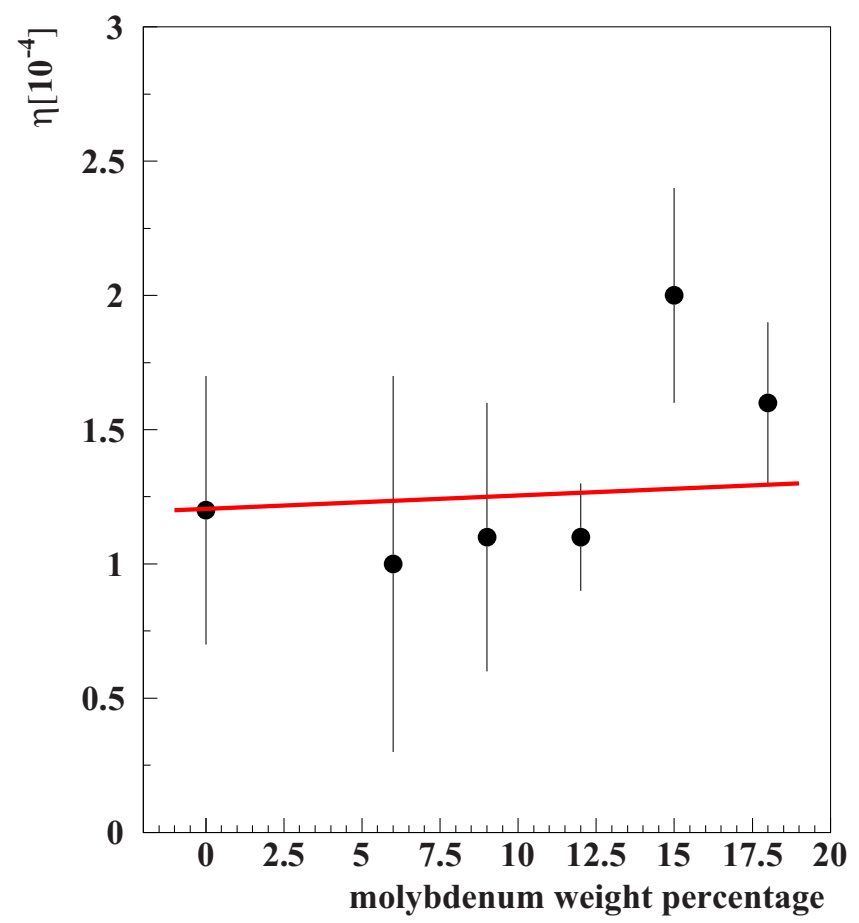

FIG. 22. Loss parameter of UCN $\eta$ over molybdenum content of the sample coating for different NiMo alloys. The line shows the theoretically expected values from $W / V_{\mathrm{f}}$. 
for molybdenum contents of 6.5 at.\% or less in NiMo alloys at room temperature. This finding may contribute to solve an ambiguity in literature; cf. Refs. [43,57,89].

The $\beta$ and $\eta$ values for the three paramagnetic NiMo alloys are small and suitable for the transport and storage of polarized UCN. Copper is also suitable for transport and storage of polarized UCN, although with a somewhat lower optical potential $V_{\mathrm{f}}$ compared to the NiMo alloys. For the dPS sample, the results were well suited for the use in a storage chamber for spin precession of UCN in an experiment to determine the electric dipole moment of the neutron. The $\beta$ and $\eta$ values of NiMo85/15 on an aluminium substrate are in fair agreement with those on glass.

As one can see from Figs. 19 and 22 and from Table III, the sample NiMo88/12 has lower losses and lower depolarization probabilities compared to the other paramagnetic NiMo samples. Also in a transmission experiment, NiMo88/12 had the best value; see Ref. [47]. Copper and dPS with similar small depolarization probabilities or losses to NiMo88/12 have much lower transmission values because of their lower optical potential.

In earlier experiments, depolarization probabilities were reported for (e.g., Ref. [71]) (i) copper (99.9\%, "Good Fellow") $\beta=(0.73 \pm 0.14) \times 10^{-5}$ and (ii) copper (a trap before graphite coating) $\beta=(1.70 \pm 0.10) \times 10^{-5}$. Our average for copper is (cf. Table III) $\beta=(0.12 \pm 0.03) \times 10^{-5}$. Spin-flip probabilities on copper were also reported in Ref. [98] as $\beta=$ $\left(0.67_{-0.25}^{+0.50}\right) \times 10^{-5}$. The data from Ref. [71] and our data show that three different copper samples give three $\beta$ values, being significantly different by 4.4 and 15.8 standard deviations, respectively. This is amazing and cannot be explained by the properties of copper alone. There must be some contribution from surface effects which are responsible for these different results. This could also be the reason for the unexpected dependence of $\beta$ over the content of molybdenum.

Following Refs. [99,100], the elastic cross section for a spin flip is due to incoherent scattering. At or in the surfaces of our samples, hydrogen is the only element with a large incoherent cross section, $\sigma_{\text {inc }}=80.3 \mathrm{~b}$ [40]. Thus, hydrogen is the main candidate for the explanation of these surface effects; see also Refs. [49,96] and Ref. [41], pp. $197 \mathrm{ff}$.

Finally, we confirm the conclusions from earlier experiments—see Ref. [37] and references therein - that all material surfaces seem to possess some common feature that is responsible for losses larger than expected from $W / V_{\mathrm{f}}$.

\section{ACKNOWLEDGMENTS}

We thank P. Fierlinger, TU Munich, for help with the DAQ system and R. Eichler ETH Zürich for fruitful discussions. This experiment benefited from the excellent technical support by F. Burri, D. George, U. Heidelberger, A. Hirt, M. Horvath, Ph. Kaufmann, M. Meier, M. Negrazus, P. Rüttimann, M. Sibold, R. Voegeli, V. Vrankovic (all PSI), and Th. Brenner and R. Bebb from the ILL. The kind hospitality of ILL is gratefully acknowledged.
[1] L. Landau, Zh. Eksp. Teor. Fiz. 32, 405 (1957) [Sov. Phys. JETP 5, 336 (1957)]; Nucl. Phys. 3, 127 (1957).

[2] E. M. Purcell and N. F. Ramsey, Phys. Rev. 78, 807 (1950).

[3] N. F. Ramsey, Phys. Rev. 109, 225 (1958).

[4] H. P. Stapp, Phys. Rev. 125, 2139 (1962).

[5] A. Sakharov, Pisma Zh. Eksp. Teor. Fiz. 5, 32 (1967) [JETP Lett. 5, 24 (1967)]; Usp. Fiz. Nauk 161, 61 (1991) [Sov. Phys. Usp. 34, 392 (1991)].

[6] D. E. Morrissey and M. J. Ramsey-Musolf, New J. Phys. 14, 125003 (2012).

[7] I. Altarev, G. Ban, G. Bison, K. Bodek, M. Burghoff, M. Cvijovic, M. Daum, P. Fierlinger, E. Gutsmiedl, G. Hampel et al., Nucl. Instrum. Methods Phys. Res. A 611, 133 (2009).

[8] C. A. Baker, G. Ban, K. Bodek, M. Burghoff, Z. Chowdhuri, M. Daum, M. Fertl, B. Franke, P. Geltenbort, K. Green et al., Phys. Proc. 17, 159 (2011).

[9] P. G. Harris, C. A. Baker, K. Green, P. Iaydjiev, S. Ivanov, D. J. R. May, J. M. Pendlebury, D. Shiers, K. F. Smith, M. van der Grinten, and P. Geltenbort, Phys. Rev. Lett. 82, 904 (1999).

[10] C. A. Baker, D. D. Doyle, P. Geltenbort, K. Green, M. G. D. van der Grinten, P. G. Harris, P. Iaydjiev, S. N. Ivanov, D. J. R. May, J. M. Pendlebury et al., Phys. Rev. Lett. 97, 131801 (2006).

[11] J. M. Pendlebury, S. Afach, N. J. Ayres, C. A. Baker, G. Ban, G. Bison, K. Bodek, M. Burghoff, P. Geltenbort, K. Green et al., Phys. Rev. D 92, 092003 (2015).
[12] E. Fermi, Ricerca Scientifica 7, 13 (1936), see also E. Fermi, in Note e Memorie (F. N. M.), edited by E. Segrè et al. (Accademia Nazionale dei Lincei and University of Chicago Press, Rome and Chicago, 1962), Vol. I, pp. 943-979.

[13] E. Fermi and W. N. Zinn, Phys. Rev. 70, 103 (1946).

[14] E. Fermi and L. Marshall, Phys. Rev. 71, 666 (1947).

[15] A. Anghel, F. Atchison, B. Blau, B. van den Brandt, M. Daum, R. Doelling, M. Dubs, P.-A. Duperrex, A. Fuchs, D. George et al., Nucl. Instrum. Methods Phys. Res. A 611, 272 (2009).

[16] B. Lauss, Phys. Proced. 51, 98 (2014).

[17] B. Lauss, AIP Conf. Proc. 1441, 576 (2012).

[18] B. Lauss, Hyperfine Interact. 211, 21 (2012).

[19] G. Bison, M. Daum, K. Kirch, B. Lauss, D. Ries, P. SchmidtWellenburg, G. Zsigmond, T. Brenner, P. Geltenbort, T. Jenke et al., Phys. Rev. C 95, 045503 (2017).

[20] R. Golub and K. Böning, Z. Phys. B 51, 95 (1983).

[21] A. Serebrov, V. Mityukhlyaev, A. Zakharov, A. Kharitonov, V. Shustov, V. Kuz'minov M. Lasakov, R. Tal'daev, A. Aldushchenkov, V. Varlamov et al., Nucl. Instrum. Methods Phys. Res. A 440, 658 (2000).

[22] C.-Y. Liu, A. R. Young, and S. K. Lamoreaux, Phys. Rev. B 62, R3581 (2000).

[23] A. P. Serebrov, E. A. Kolomenski, M. S. Lasakov, V. A. Mityukhlyaev, A. N. Pirozhkov, I. A. Potapov, V. E. Varlamov, A. V. Vasiliev, A. R. Young, and A. A. Zakharov, JETP Lett. 74, 302 (2001). 
[24] C. L. Morris, J. M. Anya, T. J. Bowles, B. W. Fillipone, P. Geltenbort, R. E. Hill, M. Hino, S. Hoedl, G. E. Hogan, T. M. Ito et al., Phys. Rev. Lett. 89, 272501 (2002).

[25] A. Saunders, J. M. Anaya, T. J. Bowles, B. W. Filippone, P. Geltenbort, R. E. Hill, M. Hino, S. Hoedl, G. E. Hogan, T. M. Ito et al., Phys. Lett. B 593, 55 (2004).

[26] F. Atchison, B. van den Brandt, T. Bryś, M. Daum, P. Fierlinger, P. Hautle, R. Henneck, S. Heule, M. Kasprzak, K. Kirch et al., Phys. Rev. C 71, 054601 (2005).

[27] F. Atchison, B. van den Brandt, T. Bryś, M. Daum, P. Fierlinger, P. Hautle, R. Henneck, K. Kirch, J. Kohlbrecher, G. Kühne et al., Phys. Rev. Lett. 94, 212502 (2005).

[28] F. Atchison, B. Blau, B. van den Brandt, T. Bryś, M. Daum, P. Fierlinger, P. Hautle, R. Henneck, S. Heule, K. Kirch et al., Phys. Rev. Lett. 95, 182502 (2005).

[29] F. Atchison, B. Blau, K. Bodek, B. van den Brandt, T. Bryś, M. Daum, P. Fierlinger, A. Frei, P. Geltenbort, P. Hautle et al., Phys. Rev. Lett. 99, 262502 (2007).

[30] I. Altarev, F. Atchison, M. Daum, A. Frei, E. Gutsmiedl, G. Hampel, F. J. Hartmann, W. Heil, A. Knecht, J. V. Kratz et al., Phys. Rev. Lett. 100, 014801 (2008).

[31] F. Atchison, B. Blau, K. Bodek, B. van den Brandt, T. Bryś, M. Daum, P. Fierlinger, A. Frei, P. Geltenbort, P. Hautle et al., Phys. Rev. Lett. 101, 189902 (2008).

[32] I. Altarev, M. Daum, A. Frei, E. Gutsmiedl, G. Hampel, F. J. Hartmann, W. Heil, A. Knecht, J. V. Kratz, T. Lauer et al., Eur. Phys. J. A 37, 9 (2008).

[33] F. Atchison, B. Blau, K. Bodek, B. van den Brandt, T. Bryś, M. Daum, P. Fierlinger, A. Frei, P. Geltenbort, P. Hautle et al., Nucl. Instrum. Methods Phys. Res. A 611, 252 (2009).

[34] A. P. Serebrov, M. S. Lasakov, A. K. Fomin, P. Geltenbort, A. N. Murashkin, I. A. Krasnoshekova Yu. P. Rudnev, and A. V. Vasiliev, Nucl. Instrum. Methods Phys. Res. A 545, 490 (2005).

[35] S. Afach, G. Ban, G. Bison, K. Bodek, Z. Chowdhuri, M. Daum, M. Fertl, B. Franke, P. Geltenbort, Z. D. Grujić et al., Eur. Phys. J. A 51, 143 (2015).

[36] G. Ban, G. Bison, K. Bodek, Z. Chowdhuri, P. Geltenbort, W. C. Griffith, V. Hélaine, R. Henneck, M. Kasprzak, Y. Kermaidic et al., Eur. Phys. J. A 52, 326 (2016).

[37] R. Golub, D. J. Richardson, and S. K. Lamoreaux, Ultra-Cold Neutrons (Adam Hilger, Bristol, Philadelphia, New York, 1991).

[38] M. Hainbuchner and E. Jericha, Bound coherent neutron scattering lenghts from periodic table switchboard, Atominstitut der Österreichischen Universitäten, A-1020-Wien, Austria (2001), http://www.ati.ac.at/ neutropt/scattering/table.html.

[39] www.webelements.com.

[40] NIST, National Institute of Standards and Technology, https://www.ncnr.nist.gov/resources/n-lengths/.

[41] V. K. Ignatovich, The Physics of Ultracold Neutrons, Oxford Series of Neutron Scattering in Condensed Matter (Clarendon Press, Oxford, 1990).

[42] B. Lauss (private communication).

[43] M. Shiga, in 3d, 4d and 5d Elements, Alloys and Compounds, Landolt-Börnstein - Group III Condensed Matter 19A, edited by H. P. J. Wijn (Springer-Verlag, Berlin, Heidelberg, 1986), p. 563.

[44] F. Acker and R. Huguenin, J. Phys. F 6, L147 (1976).

[45] J. W. Cable, M. R. Khan, G. P. Felcher, and Ivan K. Schuller, Phys. Rev. B 34, 1643 (1986).
[46] F. Atchison, B. Blau, M. Daum, P. Fierlinger, P. Geltenbort, M. Gupta, R. Henneck, S. Heule, M. Kasprzak, A. Knecht et al., Nucl. Instrum. Methods Phys. Res. B 260, 647 (2007).

[47] M. Daum, B. Franke, P. Geltenbort, E. Gutsmiedl, S. Ivanov, J. Karch, M. Kasprzak, K. Kircha, A. Kraft, T. Lauer et al., Nucl. Instrum. Methods Phys. Res. A 741, 71 (2014).

[48] F. Atchison, T. Bryś, M. Daum, P. Fierlinger, P. Geltenbort, R. Henneck, S. Heule, M. Kasprzak, K. Kirch, A. Pichlmaier et al., Phys. Lett. B 625, 19 (2005).

[49] F. Atchison, T. Bryś, M. Daum, P. Fierlinger, P. Geltenbort, R. Henneck, S. Heule, M. Kasprzak, K. Kirch, A. Pichlmaier et al., Phys. Rev. C 76, 044001 (2007).

[50] V. V. Nesvizhevsky, A. V. Strelkov, P. Geltenbort, and P. S. Iaydjiev, Eur. Phys. J. AP 6, 151 (1999).

[51] V. K. Ignatovich and M. Utsuro, Nucl. Instrum. Methods Phys. Res. 440, 709 (2000).

[52] A. Serebrov, N. Romanenko, O. Zherebtsov, M. Lasakov, A. Vasiliev, A. Fomin, P. Geltenbort, I. Krasnoshekova, A. Kharitonov, and V. Varlamov, Phys. Lett. 335, 327 (2005).

[53] A. L. Barabanov and K. V. Protasov, Phys. Lett. A 346, 378 (2005).

[54] Yu. Sobolev, Th. Lauer, Yu. Borisov, M. Daum, N. du Fresne, L. Göltl, G. Hampel, W. Heil, A. Knecht, M. Keunecke et al., Nucl. Instrum. Methods Phys. Res. A 614, 461 (2010).

[55] R. Kovacs-Mezei, Th. Krist, and Zs. Révay, Nucl. Instrum. Methods Phys. Res. A 586, 51 (2008).

[56] A. Schebetov, A. Kovalev, B. Peskov, N. Pleshanov, V. Pusenkov, P. Schubert-Bischoff, G. Shmelev, Z. Soroko, V. Syromyatnikov, V. Ul'yanov, and A. Zaitsev, Nucl. Instrum. Methods Phys. Res. A 432, 214 (1999).

[57] S. Ghosh, N. Das, and A. Mookerjee, J. Phys.: Condens. Matter 10, 11773 (1998).

[58] P. Harris (private communication).

[59] K. Bodek, M. Daum, R. Henneck, S. Heule, M. Kasprzak, K. Kirch, A. Knecht, M. Kuźniak, B. Lauss, M. Meier et al., Nucl. Instrum. Methods Phys. Res. A 597, 222 (2008).

[60] M. G. D. van der Grinten, J. M. Pendlebury, D. Shiers, C. A. Baker, K. Green, P. G. Harris, P. S. Iaydjiev, S. N. Ivanov, and P. Geltenbort, Nucl. Instrum. Methods Phys. Res. A 423, 421 (1999).

[61] F. Atchison, B. Blau, M. Daum, P. Fierlinger, A. Foelske, P. Geltenbort, M. Gupta, R. Henneck, S. Heule, M. Kasprzak et al., Phys. Lett. B 642, 24 (2006).

[62] F. Atchison, T. Bryś, M. Daum, P. Fierlinger, A. Foelske, M. Gupta, R. Henneck, S. Heule, M. Kasprzak, K. Kirch et al., Diam. Relat. Mater. 16, 334 (2007).

[63] F. Atchison, B. Blau, M. Daum, P. Fierlinger, P. Geltenbort, R. Henneck, S. Heule, M. Kasprzak, K. Kirch, K. Kohlik et al., Phys. Rev. C 74, 055501 (2006).

[64] V. P. Alfimenko, A. V. Strelkov, V. N. Shvetsov, V. V. Nesvizhevskii, A. P. Serebrov, R. R. Tal'daev, and A. G. Kharitonov, Pis'ma Zh. Eksp. Teor. Fiz. 55, 92 (1992) [JETP Lett. 55, 84 (1992)].

[65] T. Bryś, M. Daum, P. Fierlinger, A. Fomin, P. Geltenbort, R. Henneck, K. Kirch, A. Kharitonov, I. Krasnoshekova, M. Kuźniak et al., Nucl. Instrum. Methods Phys. Res. A 551, 429 (2005).

[66] F. Atchison, A. Bergmaier, M. Daum, M. Döbeli, G. Dollinger, P. Fierlinger, A. Foelske, R. Henneck, S. Heule, M. Kasprzak et al., Nucl. Instrum. Methods Phys. Res. A 587, 82 (2008). 
[67] E. Korobkina, R. Golub, J. Butterworth, P. Geltenbort, and S. Arzumanov, Phys. Rev. B 70, 035409 (2004).

[68] Th. Brenner, P. Fierlinger, P. Geltenbort, E. Gutsmiedl, A. Hollering, T. Lauer, G. Petzoldt, D. Ruhstorfer, J. Schroffenegger, K. M. Seemann et al., Appl. Phys. Lett. 107, 121604 (2015).

[69] M. Kuzniak, Ph.D. thesis, Jagiellonian University, Marian Smoluchowski Institute of Physics, Nuclear Physics Department, Krakow, Poland, 2008 (unpublished).

[70] A Serebrov, A. Vasiliev, M. Lasakov, Yu. Rudnev, I. Krasnoshekova, P. Geltenbort, J. Butterworth, T. Bowles, C. Morris, S. Seestrom et al., Nucl. Instrum. Methods Phys. Res. A 440, 717 (2000).

[71] A. P. Serebrov, M. S. Lasakov, A. V. Vassiljev, I. A. Krasnoschekova, Yu. P. Rudnev, A. K. Fomin, V. E. Varlamov, P. Geltenbort, J. Butterworth, A. R. Young, and U. Pesavento, Phys. Lett. A 313, 373 (2003).

[72] Yu. Pokotilovski, JETP Lett. 76, 131 (2002).

[73] T. Bryś, M. Daum, P. Fierlinger, P. Geltenbort, D. George, M. Gupta, R. Henneck, S. Heule, M. Horvat, M. Kasprzak et al., Nucl. Instrum. Methods Phys. Res. A 550, 637 (2005).

[74] V. V. Vladimirskii, Sov. Phys. JETP 12, 740 (1961).

[75] Yu. G. Abov, V. V. Vasil'ev, V. V. Vladimirskii, and I. B. Rozhnin, Pis'ma Zh. Eksp. Teor. Fiz. 44, 369 (1986) [JETP Lett. 44, 369 (1986)].

[76] M. Daum, P. Fierlinger, B. Franke, P. Geltenbort, L. Goeltl, E. Gutsmiedl, J. Karch, G. Kessler, K. Kirch, H.-C. Koch et al., Phys. Lett. B 704, 456 (2011).

[77] R. Holsinger and K. Halbach, POISSON SUPERFISH code, Regents of the University of California, http://laacg.lanl.gov/laacg/services/download_sf.phtml.

[78] Merz Hygiene GmbH, 60048 Frankfurt am Main, www.merzhygiene.de.

[79] ILL YellowBook, www.ill.fr/YellowBook/PF2/.

[80] W. Drexel, Neutron News 1, 23 (1990).

[81] A. Steyerl, H. Nagel, F.-X. Schreiber, K.-A. Steinhauser, R. Gähler, W. Gläser, P. Ageron, J. M. Astruc, W. Drexel, G. Gervais, and W. Mampe, Phys. Lett. A 116, 347 (1986).

[82] P. Fierlinger, Ph.D. thesis, University of Zürich, Zürich, Switzerland, 2005 (unpublished).

[83] F. Atchison, T. Bryś, M. Daum, P. Fierlinger, A. Fomin, R. Henneck, K. Kirch, M. Kuźniak, and A. Pichlmaier, Nucl. Instrum. Methods Phys. Res. A 552, 513 (2005).
[84] S. J. Seestrom, E. R. Adamek, D. Barlow, L. J. Broussard, N. B. Callahan, S. M. Calyton, C. Cude-Woods, S. Currie, E. B. Dees et al., Phys. Rev. C 92, 065501 (2015).

[85] G. Zsigmond (private communication).

[86] G. Kessler (private communication).

[87] E. Gutsmiedl (private communication).

[88] Yu. Sobolev (private communication).

[89] P. Singh, R. Banerjee, M. Rahaman, A. V. Ruban, B. Sanyal, and A. Mookerjee, PRAMANA J. Phys. 76, 639 (2011).

[90] Particle P. D. Group, K. A. Olive, K. Agashe, C. Amsler, M. Antonelli, J.-F. Arguin, D. M. Asner, H. Baer, H. R. Band, R. M. Barnett, T. Basaglia et al., Chin. Phys. C 38, 1380 (2014).

[91] S. Arzumanov, L. Bondarenko, S. Chernyavsky, W. Drexel, A. Fomina, P. Geltenbort, V. Morozov, Yu. Panin, J. Pendlebury, and K. Schreckenbach, Phys. Lett. B 483, 15 (2000).

[92] A. Serebrov, V. Varlamov, A. Kharitonov, A. Fomin, Yu. Pokotilovski, P. Geltenbort, J. Butterworth, I. Krasnoschekova, M. Lasakov, R. Tal'daev et al., Phys. Lett. B 605, 72 (2005).

[93] A. P. Serebrov, V. E. Varlamov, A. G. Kharitonov, A. K. Fomin, Yu. N. Pokotilovski, P. Geltenbort, I. A. Krasnoschekova, M. S. Lasakov, R. R. Tal'daev, A. V. Vassiljev, and O. M. Zherebtsov, Phys. Rev. C 78, 035505 (2008).

[94] A. Pichlmaier, V. Varlamov, K. Schreckenbach, and P. Geltenbort, Phys. Lett. B 693, 221 (2010).

[95] S. Arzumanov, L. Bondarenko, S. Chernyavsky, P. Geltenbort, V. Morozov, V.V. Nesvizhevsky, Yu. Panina, and A. Strepetov, Phys. Lett. B 745, 79 (2015).

[96] W. Mampe, P. Ageron, and R. Gähler, Z. Phys. B 45, 1 (1981).

[97] A. P. Serebrov, J. Butterworth, M. Daum, A. K. Fomin, P. Geltenbort, K. Kirch, I. A. Krasnoschekova, M. S. Lasakov, Yu. P. Rudnev, V. E. Varlamov, and A. V. Vassiljev, Phys. Lett. A 309, 218 (2003).

[98] Z. Tang, E. R. Adamek, A. Brandt, N. B. Callahan, S. M. Clayton, S. A. Currie, T. M. Ito, M. Makela Y. Masuda, C. L. Morris et al., Nucl. Instrum. Methods Phys. Res. A 827, 32 (2016).

[99] I. I. Gurevich and L. V. Tarasov, Low-Energy Neutron Physics (North-Holland, Amsterdam, 1968).

[100] V. F. Turchin, Slow Neutrons (Israel Program for Scientific Translations, Jerusalem, 1965). 